\title{
BMJ Open Protocol for the Electroencephalography Guidance of Anesthesia to Alleviate Geriatric Syndromes (ENGAGES) study: a pragmatic, randomised clinical trial
}

T S Wildes, ${ }^{1}$ A C Winter, ${ }^{2}$ H R Maybrier, ${ }^{1}$ A M Mickle, ${ }^{1}$ E J Lenze, ${ }^{3}$ S Stark, ${ }^{4,5}$ N Lin, ${ }^{6}$ S K Inouye, ${ }^{7,8}$ E M Schmitt, ${ }^{8}$ S L McKinnon, ${ }^{1}$ M R Muench, ${ }^{1}$ M R Murphy, ${ }^{1}$ R T Upadhyayula, ${ }^{1}$ B A Fritz, ${ }^{1}$ K E Escallier, ${ }^{1}$ G P Apakama, ${ }^{1}$ D A Emmert, ${ }^{1}$ T J Graetz, ${ }^{1}$ T W Stevens, ${ }^{1}$ B J Palanca, ${ }^{1}$ R L Hueneke, ${ }^{1}$ S Melby, ${ }^{9}$ B Torres, ${ }^{1}$ $\mathrm{J}$ Leung, ${ }^{10} \mathrm{E}$ Jacobsohn, ${ }^{11}$ M S Avidan ${ }^{1}$

To cite: Wildes TS, Winter AC, Maybrier HR, et al. Protocol for the Electroencephalography Guidance of Anesthesia to Alleviate Geriatric Syndromes (ENGAGES) study: a pragmatic, randomised clinical trial. BMJ Open 2016;6:e011505. doi:10.1136/bmjopen-2016011505

- Prepublication history and additional material is available. To view please visit the journal (http://dx.doi.org/ 10.1136/bmjopen-2016011505).

Received 17 February 2016 Revised 3 May 2016 Accepted 19 May 2016

CrossMark

For numbered affiliations see end of article.

Correspondence to Dr M S Avidan; avidanm@anest.wustl.edu

\section{ABSTRACT}

Introduction: Postoperative delirium, arbitrarily defined as occurring within 5 days of surgery, affects up to $50 \%$ of patients older than 60 after a major operation. This geriatric syndrome is associated with longer intensive care unit and hospital stay, readmission, persistent cognitive deterioration and mortality. No effective preventive methods have been identified, but preliminary evidence suggests that EEG monitoring during general anaesthesia, by facilitating reduced anaesthetic exposure and EEG suppression, might decrease incident postoperative delirium. This study hypothesises that EEGguidance of anaesthetic administration prevents postoperative delirium and downstream sequelae, including falls and decreased quality of life.

Methods and analysis: This is a 1232 patient, blockrandomised, double-blinded, comparative effectiveness trial. Patients older than 60 , undergoing volatile agentbased general anaesthesia for major surgery, are eligible. Patients are randomised to 1 of 2 anaesthetic approaches. One group receives general anaesthesia with clinicians blinded to EEG monitoring. The other group receives EEG-guidance of anaesthetic agent administration. The outcomes of postoperative delirium ( $\leq 5$ days), falls at 1 and 12 months and health-related quality of life at 1 and 12 months will be compared between groups. Postoperative delirium is assessed with the confusion assessment method, falls with ProFaNE consensus questions and quality of life with the Veteran's RAND 12-item Health Survey. The intentionto-treat principle will be followed for all analyses. Differences between groups will be presented with 95\% Cls and will be considered statistically significant at a two-sided $p<0.05$.

Ethics and dissemination: Electroencephalography Guidance of Anesthesia to Alleviate Geriatric Syndromes (ENGAGES) is approved by the ethics board at Washington University. Recruitment began in January 2015. Dissemination plans include presentations at scientific conferences, scientific publications, internetbased educational materials and mass media.

Trial registration number: NCT02241655; Pre-results.

\section{Strengths and limitations of this study}

- The Electroencephalography Guidance of Anesthesia to Alleviate Geriatric Syndromes (ENGAGES) study is a pragmatic clinical trial, conducted in a real world clinical setting.

- The electroencephalography-guided anaesthetic protocol is straightforward and inexpensive; it would be feasible to disseminate and implement broadly.

- The effectiveness of the electroencephalographyguided anaesthetic protocol will depend on clinicians' adherence to the protocol.

- As delirium is a fluctuating disorder, it may occasionally be missed despite rigorous and validated assessment methods.

- Some patients might be unable to speak in the early postoperative period (eg, have a tracheal tube in place), which will curtail the sensitivity of delirium assessment.

\section{BACKGROUND}

Within the next 40 years, $>110$ million Americans will exceed the age of $60,{ }^{1}$ and many of them $(>40 \%)$ will require elective surgery. $^{2}$ The geriatric syndrome of postoperative delirium is one of the most common complications observed with the physiological stress of major surgery and anaesthesia. It affects up to $70 \%$ of surgical patients older than 60, with most studies showing an incidence of 30-50\%. ${ }^{3}$ Delirium is an acute and fluctuating neurologic disorder that reflects a change from baseline cognition and is characterised by the cardinal features of inattention and disorganised thinking. ${ }^{4}$ Postoperative delirium typically first manifests between 24 and 96 hours following the surgical intervention. While it is unclear why postoperative delirium occurs so 
frequently, consistently described risk factors for delirium include older age, male sex, mild cognitive impairment, dementia, sensory impairment and chronic medical illness. $^{5}$

Postoperative delirium has substantial implications at a societal level for healthcare professionals and for individual patients and their families. It is estimated that delirium is associated with additional healthcare costs exceeding US\$60 000 per patient per year. ${ }^{6}$ The occurrence and the duration of delirium are linked with increased morbidity and mortality, prolonged length of hospital and intensive care unit (ICU) stay, as well as functional and cognitive decline necessitating nursing home or long-term care facility placement. ${ }^{7-10}$ Preoperative surveys completed by 1000 patients at our institution, Barnes-Jewish Hospital, a tertiary care facility at Washington University in St. Louis, showed that $~ 40 \%$ of surgical patients highlight postoperative delirium (or acute confusion) as one of their top concerns, and $30 \%$ of all patients are worried that they will still have problems thinking normally when they return home to recover. Another survey study showed that when in-hospital delirium occurs patients' family members are deeply affected by the acute neurologic deterioration and the impact upon recovery. ${ }^{11}$

Delirious patients are unable to participate effectively in rehabilitation and are therefore susceptible to other postoperative geriatric syndromes and adverse events, including falls, pressure ulcers, functional decline, pneumonia, hospital readmission and discharge to a nursing home or extended care facility. ${ }^{8}{ }^{12-16}$ There is even evidence that patients who have periods of delirium while in hospital may continue to experience persistent delirium after going home, and among these patients, the risks of mortality, institutionalisation and functional and cognitive decline are even worse than those patients who experienced delirium but recovered. ${ }^{17}$ Patients who experience postoperative delirium report persistently decreased quality of life. ${ }^{8}$ Furthermore, additional studies suggest that incidence and duration of delirium may be associated with long-term postoperative cognitive dysfunction. ${ }^{18}{ }^{19}$ It is therefore a public health priority to test plausible interventions to prevent, identify and treat postoperative delirium.

Even though postoperative delirium is a pressing healthcare concern, there are barriers to making progress in its prevention and treatment. Delirium is difficult to diagnose as most patients with delirium are hypoactive or lethargic ${ }^{20}$ while medical staff typically recognise delirium when patients are hyperactive and agitated. Hypoactive characteristics may also be easily regarded as a normal phenotype in a patient recovering from surgery or general anaesthesia. Furthermore, no group of healthcare practitioners involved in the direct surgical care of patients has taken ownership of delirium as a priority needing their attention. It is not currently standard of care to routinely assess surgical patients for delirium, approaches for preventing postoperative delirium have not been applied to surgical patients and treatment options for delirium are limited. Delirium is a common complication of surgery and anaesthesia with serious consequences for patients and their families, yet it remains an orphan problem, and no effective prophylactic or curative treatments for postoperative delirium have been identified.

Detecting delirium routinely in surgical patients using a validated and practical approach like the confusion assessment method $(\mathrm{CAM})^{21}$ could allow target therapies and potentially improve outcomes. For example, the Hospital Elder Life Program (HELP) ${ }^{22}{ }^{23}$ has been demonstrated to be effective for prevention of postoperative delirium, and principles and protocols from this programme will be used in the proposed study. In addition, although the effectiveness of the Acute Care for Elders model has not yet been evaluated in the postoperative setting, delirious patients could be targeted to receive components of this model (frequent medical review, early rehabilitation, early discharge planning, prepared environment, patientcentred care), all of which have been shown to decrease geriatric syndromes, such as falls, in vulnerable patients. ${ }^{24}{ }^{25}$ Identifying and if possible preventing delirium in surgical patients might present an important opportunity to improve numerous outcomes beyond a reduction in the delirium burden.

Although it is very likely that anaesthetic management contributes to the occurrence of postoperative delirium, to date there are no validated anaesthetic approaches to preventing delirium. Four randomised, controlled studies in diverse surgical settings have suggested a decrease in postoperative delirium with bispectral index (BIS) guidance of general anaesthesia. ${ }^{26-29}$ The BIS is one of several proprietary EEG indices of anaesthetic depth, on the basis of EEG waveform processing, with numbers approaching 100 suggesting arousal or wakefulness, and numbers approaching 0 reflecting absent detectable brain electrical activity. ${ }^{30} \mathrm{~A}$ meta-analysis of these four randomised controlled trials showed that EEG (or BIS) guidance of anaesthesia was associated with a marked reduction in postoperative delirium with a pooled OR of 0.56 ( $95 \%$ CI 0.42 to 0.73 , heterogeneity $\mathrm{p}$ value $=0.54){ }^{29}$ Also of interest are several studies that have examined the relationship between low intraoperative BIS values and intermediate term postoperative mortality. ${ }^{31-35}$ Building on these a study has demonstrated that intraoperative EEG burst suppression specifically, especially when coinciding with hypotension, is associated with increased 90 -day postoperative mortality. ${ }^{36}$

Despite the findings from these studies and recommendations from the National Institute for Health and Care Excellence in the UK that electroencephalography guidance of anaesthesia should be routine for vulnerable patients, ${ }^{37}$ intraoperative EEG monitoring has not become standard anaesthetic practice, and there is ongoing controversy about the utility of electroencephalography guidance of anaesthesia. ${ }^{38}$ For example, in the UK only $2 \%$ of anaesthesia practitioners routinely 
incorporate EEG monitoring in their practice, ${ }^{39}$ and it is possible that adoption is similarly low in the USA. The results of several clinical trials have led anaesthesia practitioners to question whether EEG-guidance meaningfully changes anaesthetic administration in real world settings, ${ }^{40-42}$ and the mechanisms by which EEG-guidance could decrease postoperative delirium have not been clarified. In the USA, the American Society of Anesthesiologists in its most recent guidelines on brain monitoring does not recommend EEG monitoring as standard care for any patient population, procedure or anaesthetic technique. ${ }^{43}$ A pragmatic, randomised clinical trial would address this controversy and could help to inform the standard of care going forward. The plausibility for EEG guidance preventing postoperative delirium is that it might help practitioners to avoid excessive anaesthetic administration to vulnerable patients. ${ }^{44}$ During general anaesthesia, BIS values $<30$ are usually reflective of periods of EEG burst suppression, ${ }^{45}{ }^{46}$ which is often indicative of excessively deep anaesthesia (see figure 1). One study found a specific association between low BIS values and postoperative delirium, and the investigators hypothesised that burst suppression could be linked to postoperative delirium. ${ }^{28}$ An observational study in cardiac surgery patients reported an association between intraoperative burst suppression and postoperative delirium, ${ }^{47}$ and similarly EEG suppression in critically ill patients reportedly predicts postcoma delirium. ${ }^{48}$ EEG burst suppression and low BIS values have sometimes been shown to be associated with intermediate term mortality after surgery and critical illness. ${ }^{31-36} 4950$ The proposed Electroencephalography Guidance of Anesthesia to Alleviate Geriatric Syndromes (ENGAGES) study is designed as a parallel group, pragmatic, superiority trial to test whether a simple EEG-guided protocol, designed to minimise epochs of low BIS values and EEG burst suppression, prevents postoperative delirium as well as its downstream public health sequelae, such as deterioration

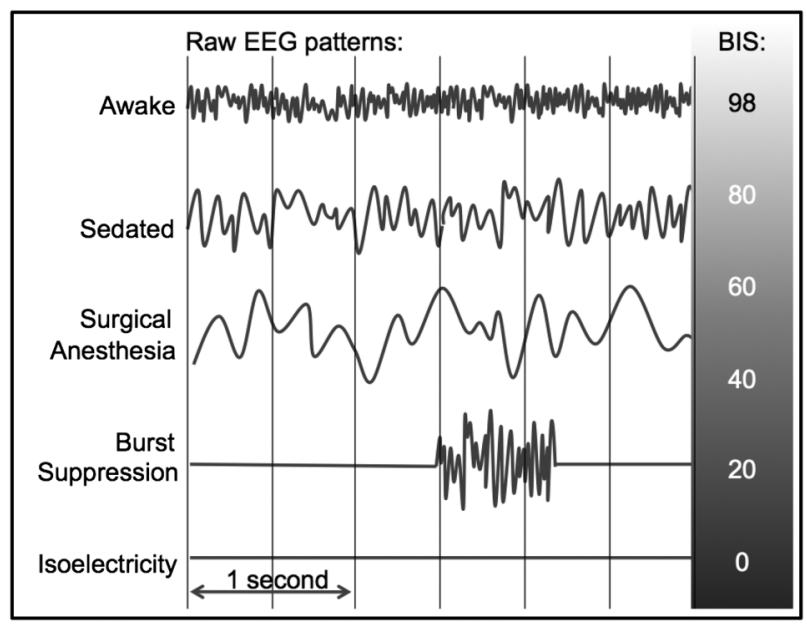

Figure 1 Stylized common EEG patterns from frontal EEG channel seen with progressively increasing anaesthetic depth. BIS, bispectral index. in health-related quality of life and injurious falls. The ENGAGES study has three main hypotheses: (1) EEG-guidance of anaesthesia is effective in preventing delirium; (2) through prevention of delirium, EEG-guided anaesthesia prevents postoperative falls and improves patient-reported quality of life and (3) providing a targeted safety intervention will prevent postoperative falls.

\section{METHODS}

\section{Research design overview}

The Human Research Protection Office at Washington University School of Medicine has approved the study. This protocol, which details the design of the ENGAGES study, includes all the elements elaborated in the SPIRIT (Standard Protocol Items: Recommendations for Interventional Trials) checklist. $^{51}{ }^{52}$ The ENGAGES study will be a pragmatic randomised clinical trial enrolling 1232 patients 60 years and older who will undergo elective major surgery at Barnes Jewish Hospital, St. Louis, Missouri, USA. This hospital is an academic medical centre in the Midwestern USA, which is affiliated to Washington University School of Medicine and serves a diverse range of patients in St. Louis and its environs. Eligible patients will often be recruited through the Center for Preoperative Assessment and Planning (CPAP) clinic at Barnes Jewish Hospital. Surgical patients might also be enrolled on hospital wards prior to their surgery. Participants will be randomly assigned to receive the electroencephalography-guided protocol or routine care. Assessments will be conducted at baseline, in the postoperative period during the hospital stay, at 30 days and at 1-year postsurgery. The primary outcome measure will be the incidence of postoperative delirium. During the 1-year follow-up period, health-related quality of life information and information on incident falls will be collected. At Washington University, surgical patients have been enrolled in the Systematic Assessment and Targeted Improvement of Services Following Yearly Surgical Outcomes Surveys (SATISFY-SOSNCT02032030) study since 2012. For the exploratory aim 3, there will be a prospective comorbidity-matched cohort study using the ENGAGES clinical trial population and reference subjects from the ongoing SATISFY-SOS study (see figure 2). There is ongoing rolling enrolment of participants to the SATISFY-SOS study, and information on patients is continuously being collected, updated and stored in a SQL Server database (Microsoft, Redmond, Washington, USA) hosted by the Institute of Quality Improvement, Research and Informatics at Washington University.

\section{Study subjects}

This study proposes to enrol 1232 patients who are already enrolled in the SATISFY-SOS study. Patients 60 years old and older, who are competent to provide 


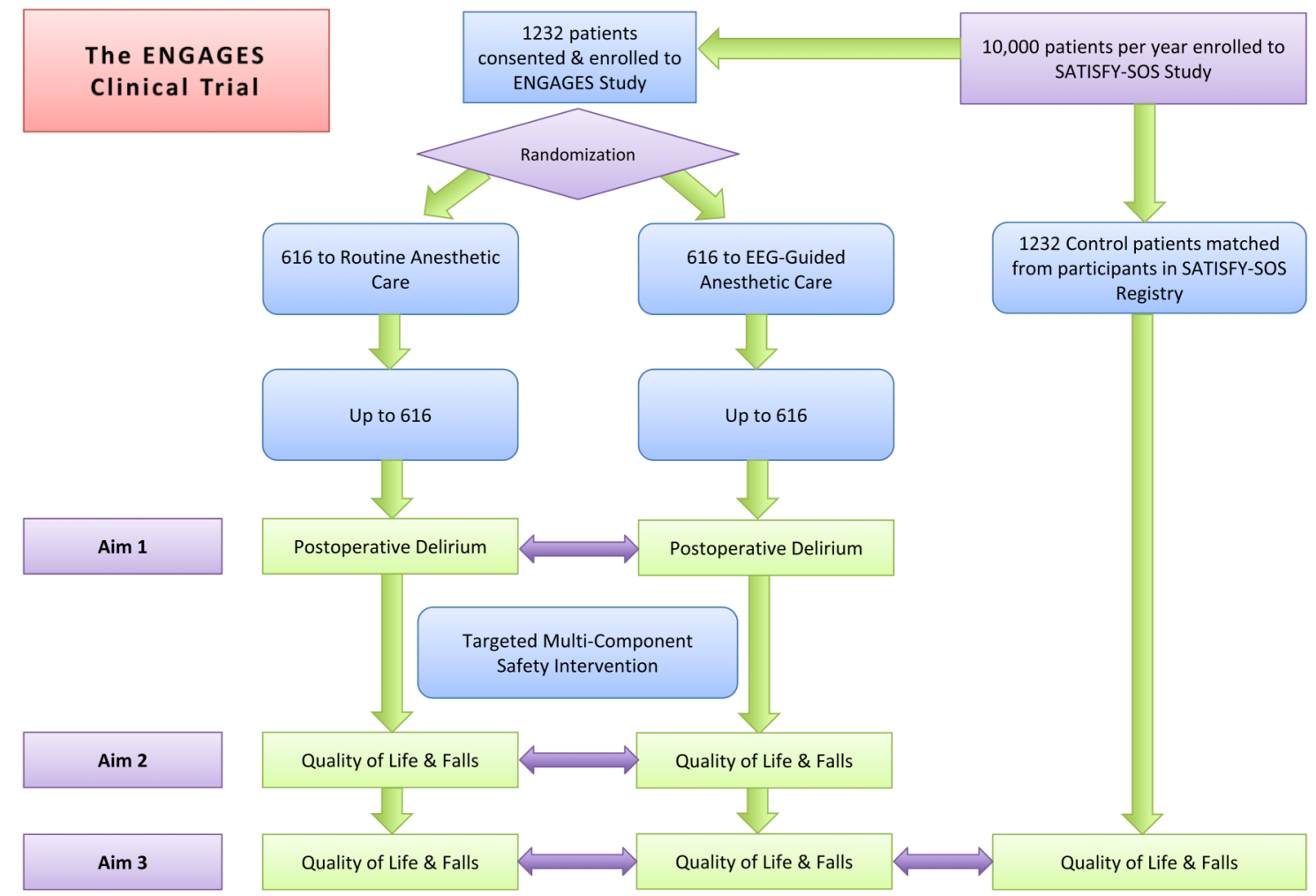

Figure 2 Flow diagram showing design overview for ENGAGES study.

informed consent and who are undergoing major elective surgery under general anaesthesia with a potent volatile anaesthetic agent that requires a minimum stay of 2 days postoperatively (eg, open cardiac surgery, open thoracic surgery, major vascular surgery, intra-abdominal surgery, open gynaecologic surgery, open urologic surgery, major orthopaedic surgery, open hepato-biliary surgery and major ear, nose and throat surgery), will be eligible for inclusion. As there are no absolute contraindications to EEG monitoring, the ENGAGES study is designed as a practical trial that will have minimal exclusions and therefore maximum applicability. Neurosurgical procedures will be excluded as surgery on the brain can confound the outcome (postoperative delirium). We will also exclude patients with preoperative delirium and patients who are unable to participate adequately in delirium screening including those who are blind, deaf or illiterate or not fluent in English. Patients with a history of intraoperative awareness during intended general anaesthesia will also be excluded. ${ }^{53}$ Patients will be excluded if, prior to their index surgery, a second surgery is planned to occur within 5 days after the index surgery. Figure 3 outlines the flow of participants in the ENGAGES study.

\section{Recruitment}

All patients will provide written informed consent for the study. Patients will often be recruited through the CPAP clinic at Barnes Jewish Hospital, St. Louis, Missouri. The majority of adults undergoing surgery at
Barnes-Jewish Hospital, about 30000 patients per year, are evaluated at CPAP. This clinic is staffed by anaesthesiologists specialising in perioperative medicine and nurses who aim to evaluate each surgical patient's perioperative risks. On average, the time frame between study enrolment at the CPAP clinic and elective surgery will be 1 week. Surgical patients might also be enrolled on hospital wards prior to their surgery.

After the research team has established the reliability of the delirium assessments, 100 patients will be enrolled to the pilot phase of the ENGAGES trial during the first year. In years $2-4$, an accrual rate of $300-400$ patients per year is anticipated. To maximise efficiency, data from the pilot will be included in the main study. ${ }^{54}$ In this pilot cohort, practical aspects of the trial's conduct will be evaluated. These include the feasibility of enrolling adequate numbers of patients in the preoperative assessment clinic; the ability of researchers to conduct the baseline preoperative assessments; the demonstration of retrieval of complete perioperative data (including repeated measures of EEG-derived parameters) from the electronic medical record; successful daily postoperative delirium assessments until postoperative day 5 or hospital discharge (for patients who remain delirious at day 5 they will be assessed until they return to baseline or until postoperative day 10$)$; and near complete (>80\%) 30-day patient-reported outcomes data.

On the basis of data from previous large clinical trials completed at our site, we expect the study population to be largely balanced between sexes and representative of 


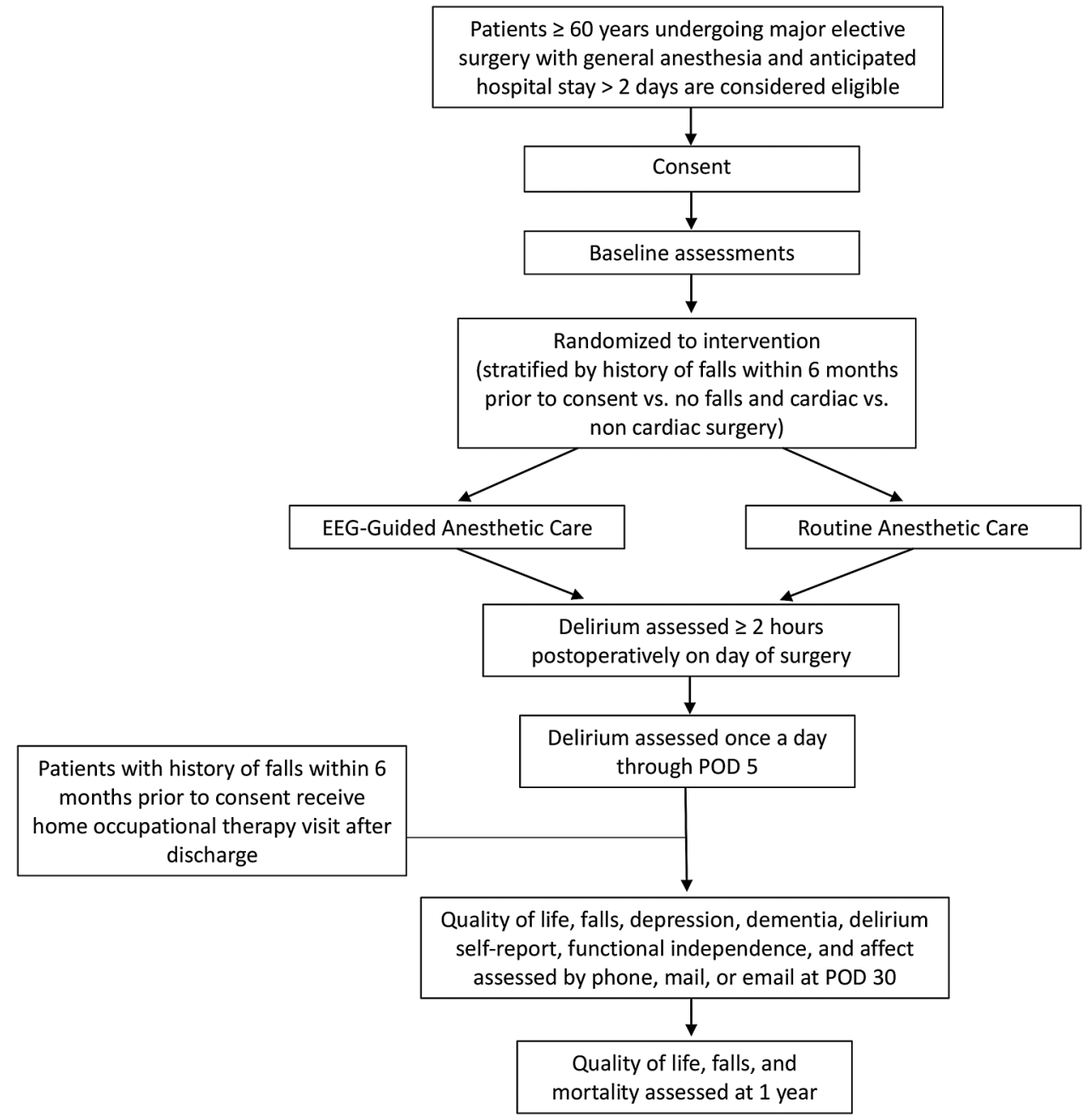

Figure 3 Flow of participants.

our environs. In two of our previous studies, we enrolled 6700 patients in St. Louis, Missouri. Results showed slightly higher enrolment of males versus females $(55 \%$ vs $45 \%$ ), and racial demographics of $\sim 80 \%$ white and $20 \%$ black or other. ${ }^{40} 41$ These results are generally representative of the population in metropolitan St. Louis and surrounding regions, where the majority of our patients reside. On the basis of 2011 census data, median household income and education levels of the population of St. Louis metropolis are representative of the national average. The ENGAGES study will enrol patients older than 60. Older patients constitute a vulnerable population and have often been underrepresented in clinical research. We anticipate that the patients enrolled in the ENGAGES study will be broadly representative of the older adult population of the USA, recognising that certain demographics (eg, Hispanic) are under-represented in St. Louis. The follow-up period after randomisation is $\sim 1$ year.

\section{Randomisation and blinding}

Randomisation will be performed at the patient level using computer-generated assignment. Eligible patients who provided written informed consent will be randomised to receive the intraoperative electroencephalography-guided protocol or routine care. To ensure that there is not major imbalance between group assignments with respect to history of falls and cardiac surgery, patients will be randomised (1:1) between the EEG-guided and routine care groups in blocks of 20 within these four strata (ie, cardiac surgery with a history of falls within 6 months, cardiac surgery without a history of falls within 6 months, noncardiac surgery with a history of falls within 6 months and non-cardiac surgery without a history of falls within 6 months). Trained members of the research team will enrol participants and will implement the assignment of participants to the EEG-guided or usual care protocols. Group assignment will be revealed to members of the anaesthetic team only when the patient enters the operating room by opening a sequentially numbered, opaque, sealed envelope in a sequence generated by one of the study's data analysts. Patients and their families will be blinded to group allocation, and different members of the research team will assist with the intervention in the operating room (will not be blinded to 
the intervention) from those conducting the postoperative assessments (will be blinded to the intervention). ${ }^{51}{ }^{52}$ To reduce predictability of a random sequence, details of patients already randomised and prior group assignments will be recorded in a separate document that is unavailable to those who enrol participants and assign interventions.

\section{Primary intervention-EEG-guided anaesthetic protocol}

The primary intervention to which patients will be randomised in this study is a pragmatic EEG-guided anaesthetic protocol (see online supplementary appendix). All anaesthesia practitioners will receive a targeted educational session on recognition of EEG patterns typically occurring during general anaesthesia. The content will be similar to that described in an article where we demonstrated that anaesthesiologists could estimate BIS (processed EEG index) values fairly accurately based on clinical context and examination of the raw EEG waveform. ${ }^{55}$ The BIS proprietary processed EEG monitor will be used for the ENGAGES study. However, the anaesthesia monitor in the operating room will be configured to display, in addition to the processed EEG index, the raw EEG waveform as well as non-proprietary EEG-derived numerical values, including the burst suppression ratio and the spectral edge frequency (figure 4). The hypothesis motivating this study is that avoidance of EEG burst suppression during anaesthesia can prevent postoperative delirium (aim 1 in figure 2) and its downstream consequences (aim 2 in figure 2). Therefore, practitioners will specifically be instructed to regularly inspect the EEG waveform for evidence of burst suppression, which is easily recognised (see figure 1). The occurrence of burst suppression is the chief trigger for decreasing anaesthetic administration in this protocol. An audible low-BIS alarm will be set at a threshold of 40 , as there is an increased likelihood of epochs of EEG burst suppression below this value. ${ }^{45}$ BIS values $<40$ will be a secondary trigger for decreasing anaesthetic administration. Importantly, the EEG-guided protocol is suggestive rather than prescriptive. Clinicians should exercise judgement and might intentionally deviate from the protocol depending on the clinical situation. In both groups, there will be an audible alarm for a low volatile anaesthetic agent (at 0.3 minimum alveolar concentration or at the clinician's discretion), which is a standard practice at our institution to prevent intraoperative awareness. BIS EEG sensors will also be applied to patients in the control group for the purpose of data comparisons between groups, but when a patient is assigned to the control arm, practitioners will be blinded to all the EEG and BIS parameters and will only see the signal quality index (SQI) of the EEG montage (figure 5). EEG monitoring may continue to be acquired via continuous recordings of EEG, eye movements and chin muscle activity for patients who are admitted to the ICU and stepdown wards or if the hospital room allows. Patients and research assistants assessing the study outcome measures (eg, delirium assessments) will be blinded to the allocated intervention.

\section{Ensure practitioner fidelity to the EEG-guided protocol}

In order for any monitor to alter clinical practice, clinicians must be able to glean useful information from the monitor and should be motivated to make decisions based on that information. One of the limitations regarding EEG guidance of anaesthesia is that teaching on electroencephalography is currently limited in anaesthesiology residencies and in nurse anaesthesia training programmes. Given this, it is unsurprising that EEG-based monitors have not been incorporated into routine anaesthetic practice. Our research group published a study showing that with a focused training session, anaesthesiologists could learn to appreciate monitor is configured for the EEG-guided arm such that the raw EEG waveform as well as the non-proprietary numerical values are displayed by the monitor, including the burst suppression ratio (SR) and the spectral edge frequency (SEF). The EEG filter is turned off so the low frequency slow delta waves (with a frequency of about $0.5 \mathrm{~Hz}$ ) are clearly visible. Turning off the filter allows EEG waves $\leq 2 \mathrm{~Hz}$ to be seen. The filter is a bandpass filter from 2 to $70 \mathrm{~Hz}$ with a notch to eliminate $60 \mathrm{~Hz}$ alternating current electrical noise. With the filter off, the system has a bandwidth of $\sim 0.25-100 \mathrm{~Hz}$.
Figure 4 The anaesthesia

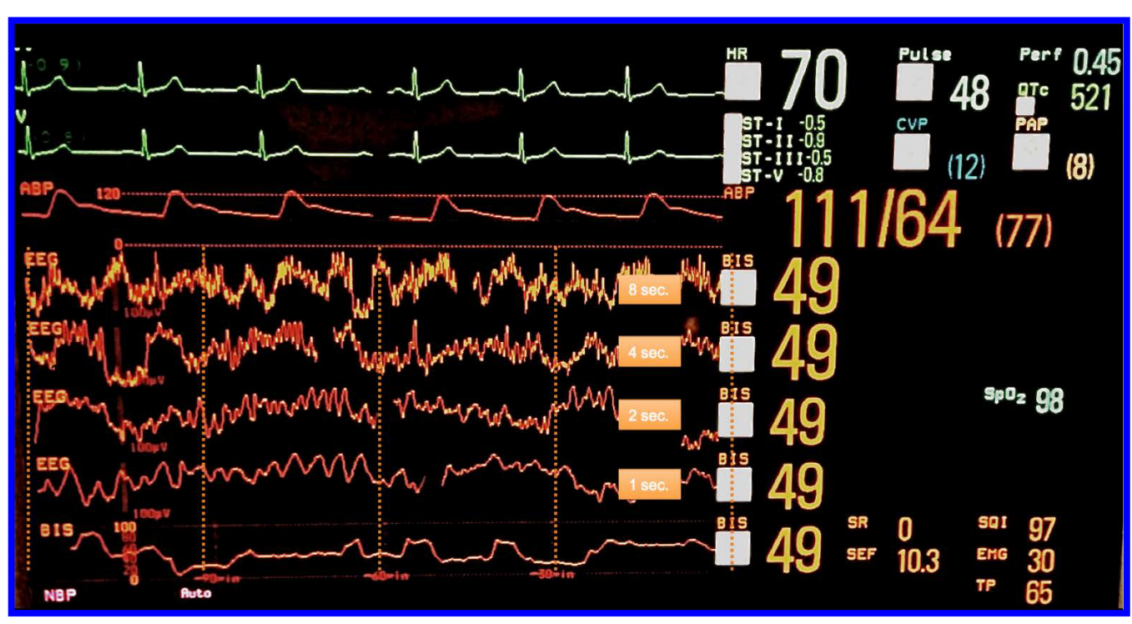


Figure 5 The anaesthesia monitor is configured for the control arm such that all the EEG and BIS parameters are hidden, and only the signal quality index (SQI) of the EEG montage is visible.

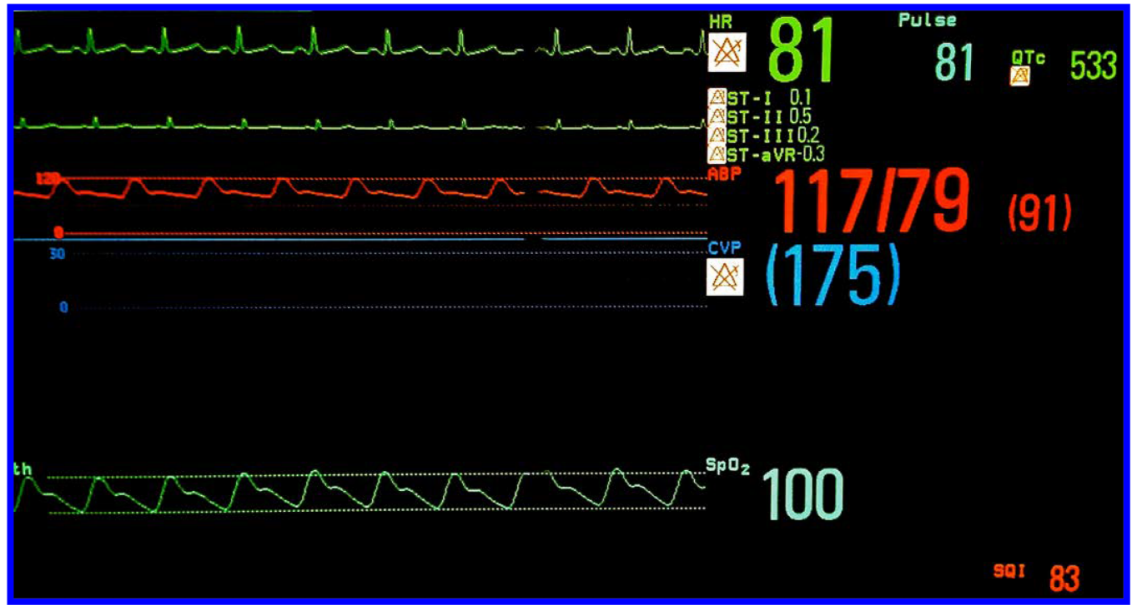

some of the key EEG changes that occur with general anaesthesia. ${ }^{55}$ We demonstrated that clinicians could learn relatively rapidly to integrate clinical context with EEG waveform information and could even accurately estimate BIS values-an index derived via computerbased processing of the raw EEG signal. ${ }^{55}$ With initiation of the ENGAGES study, we launched a training module on a non-profit international educational website, International Consortium for Electroencephalograph Training of Anesthesia Practitioners (http://www.icetap. org), titled 'EEG Waveforms and Depth of Anesthesia'. 56 Key to the success of the ENGAGES study will be educating anaesthesia practitioners at our institution about the EEG waveform and how information from the EEG can be useful in guiding anaesthetic practice. Regarding EEG-derived parameters specifically, we capture electronically proprietary (eg, BIS values) and nonproprietary (eg, burst suppression ratio) data. Therefore, we shall be able to ascertain from the phase 1 pilot study of 100 patients whether or not the EEG-guided protocol alters anaesthetic administration (eg, measured concentrations of volatile anaesthetic agents) or EEG parameters (eg, cumulative duration of EEG burst suppression). Given that the hypothesis of the ENGAGES study is that EEG guidance in the real world can alter anaesthetic management, which in turn prevents postoperative delirium, an essential proof of concept step in the pilot phase is to demonstrate our ability to alter anaesthetic practice in a range of practitioners when they use the EEG-guided protocol.

\section{Secondary intervention-multicomponent safety intervention}

On the basis of findings that multicomponent nonpharmacological protocols can improve sleep, decrease episodes of delirium and improve outcomes, 205758 the ENGAGES study will implement a multicomponent intervention including principles from the HELP ${ }^{59} 60$ for all patients enrolled in the study to attempt to prevent postdischarge falls and decrements in health-related quality of life. ${ }^{20} 575861$ These outcomes will be tracked in all patients in the ENGAGES study as they will also be enrolled in the SATISFY-SOS study. Likewise, these outcomes will be ascertained in a matched cohort of controls from the SATISFY-SOS cohort who will not be enrolled in the ENGAGES study. This will allow comparison in these outcomes between patients receiving the multicomponent safety intervention and matched controls (aim 3 in figure 2). The interventions, implemented mainly after hospital discharge, will include the following, as indicated: reduction of psychoactive drugs; advice on nonpharmacological approaches to manage sleep, anxiety and agitation; involvement of family members in care, particularly for reorientation and prevention of selfharm; encouragement of mobility and self-care; ensuring that, if needed, patients have glasses, hearing aids and dentures; home visits by occupational therapists; targeted home safety modifications; keeping patients involved in their care and communicating regularly with patients and their families. ${ }^{20}$ During the pilot phase, patients and their family members will be called and questioned about their perception of the utility of the educational resources and, if relevant, the home visit.

Preoperatively, at the time of providing informed consent for the ENGAGES study (but prior to randomisation for the primary intervention), all participants and their families will receive general information on delirium and falls derived from the HELP ${ }^{59} 60$ and from the Agency for Healthcare and Research Quality-Rand (AHRQ-Rand) hospital fall prevention programme. ${ }^{62}$ The research team will provide participants and their families with information about making the home environment safer to decrease the risk of falls and related injuries. An information sheet on improving safety in the hospital after surgery will also be provided (see online supplementary appendices: see CDC Fall Safety Information Sheet and Partners HealthCare Falls TIPS: Tips to Avoid Falls While in the Hospital). Postoperatively, prior to hospital discharge, the research staff will review participants' current medications and provide recommendations regarding any medications 
with the potential to increase the risk of falls. ${ }^{63-69}$ We will provide participants' physicians with information about any identified medications associated with fall or delirium risk (eg, strong centrally acting anticholinergics, benzodiazepines) and recommendations. ${ }^{70}$ Many of these targeted medications were highlighted in the recent American Geriatric Society Beers Criteria guidelines as potentially inappropriate medications for older persons. $^{70}$

Since a history of falls in older adults is associated with increased fall risk, ${ }^{71}$ the research staff may recommend and provide (if acceptable to patients and if patients live $<45$ miles from the hospital) home occupational therapy visits that have the general aim of improving daily activity performance/safety and prevention of falls. Since delirium in older adults is associated with increased fall risk, ${ }^{72-76}$ the research staff will recommend that patients' families exercise increased vigilance when delirium features are noted in the hospital or after hospital discharge. Patients and their families will also be reminded about the home safety assessment tool that they received at the time of enrolment (see online supplementary appendix: CDC fall safety information sheet).

\section{DATA COLLECTION}

Baseline assessment will take place at the CPAP clinic and include demographic information, a detailed medical history, physical examination, assessment of preoperative quality of life and evaluation of falls history. Delirium will be assessed daily in the postoperative period of the hospital stay. Data collected specifically for the ENGAGES study, such as the daily delirium assessments, will be entered into the Washington University School of Medicine Research Electronic Data Capture (REDCap) application. ${ }^{77}$ In the ENGAGES study, EEG data (including BIS values and EEG burst suppression durations) will be collected in the intervention group as well as in the blinded control group. ${ }^{36}{ }^{50}$ Furthermore, perioperative data (including repeated measures data) will be retrieved from the hospital's perioperative electronic medical record (Metavision by iMDsoft, Needham, Massachusetts, USA). ${ }^{36} 415378$ We are able routinely to capture high fidelity perioperative data from our MetaVision records to an SQL server (Microsoft, Redmond, Washington, USA) database. Routinely acquired data include detailed patient medical history, surgical history, specific patient risk factors, medications, Barthel Index, VR-12 quality of life data, Short Blessed Test, sleep apnoea screening, laboratory data, intraoperative medications, physiological readings and postoperative recovery parameters. Olfaction might be assessed preoperatively with the brief smell identification test, as hyposmia has been identified as a risk factor for postoperative delirium. ${ }^{79}$ All the data for SATISFY-SOS are integrated from various data sources and are stored in a single data repository housed in the Department of Anesthesiology at Washington University.

\section{Cognitive testing}

When patients are assessed for delirium, structured cognitive appraisal is performed, which gives the interviewer an opportunity to make observations that are used when scoring the CAM. In addition, as part of the routine preoperative assessment, patients will be screened for cognitive impairment with the AD-8 dementia screen $^{80}$ and with the Short Blessed Test. ${ }^{81}$ Tests from the cognitive battery of the NIH toolbox might also be incorporated in the baseline assessment. $^{82} 83$ This computer-based battery assesses Executive Function, Attention, Episodic Memory, Language, Processing Speed and Working Memory (see online supplementary appendix). Depending on time constraints, patients might complete a long form of the cognitive battery or a version focused on executive function, episodic memory and attention. Patients who prefer not to do computer-based cognitive tests will be offered paper-based cognitive tests (Trails A and B, and Stroop Color and Word Test). Impaired performance on preoperative cognitive tests is reportedly associated with postoperative delirium, ${ }^{84} 85$ and postoperative delirium has been found to be associated with persistent postoperative cognitive decline. ${ }^{18}$ Therefore, when patients are followed up postoperatively at 30 days, the Short Blessed Test will be administered on the telephone. When patients are followed up postoperatively at 1 year, the Short Blessed Test will be administered on the telephone and, if possible, follow-up cognitive assessment with the NIH toolbox will be arranged. Permission from the NIH has been obtained to use the NIH Toolbox for the ENGAGES study.

\section{Frailty assessment}

Various components of frailty are assessed routinely in the CPAP clinic and as part of the SATISFY-SOS study. These include weight loss, Charlson Comorbidity Index, individual co-morbidities, preoperative anaemia (haematocrit <35\%), functional status, 6 months history of falls and the Barthel Index. ${ }^{86}$ In addition to these measures, for the ENGAGES study we plan to measure grip strength and the Timed $\mathrm{Up}$ and Go (TUG) test. ${ }^{86}$ Grip strength will be assessed with three measurements in the dominant hand using a Jamar handheld dynamometer (Lafayette Instruments, Lafayette, Indiana, USA). ${ }^{87}$ Maximal grip strength will be selected for analysis.

\section{Patients' postoperative health and well-being}

All patients enrolled in the ENGAGES study will already have provided informed consent for the SATISFY-SOS study. Using the SATISFY-SOS infrastructure, the ENGAGES study will track postoperative patient-reported outcomes at $\sim 1$ month and at 1 year. Patient-reported outcomes tracked include health-related quality of life and postoperative falls. 


\section{Outcome measures}

Incidence of postoperative delirium

Incident delirium is the primary outcome of the ENGAGES trial (aim 1 in figure 2). A preoperative baseline assessment will be performed when patients are enrolled to participate in the ENGAGES study. Preoperative delirium is rare before elective surgery and will exclude participation in the study. Postoperative delirium assessments will be performed when patients can be aroused sufficiently to be assessed for delirium (Richmond Agitation-Sedation Scale (RASS) >-4). Patients will be assessed for delirium once daily in the afternoon/evening. Each patient will be assessed for delirium up to postoperative day 5 . Delirium will be diagnosed based on a combined approach consisting of standardised daily CAM evaluations coupled with a structured chart review. The CAM has been described as a viable tool to be used by non-psychiatrists for delirium detection. $^{21}$ It has subsequently been validated in numerous studies and has a sensitivity of $>94 \%$ and a specificity of $>89 \%$ against a reference standard. ${ }^{88}$ Trained research team members who are blinded to treatment allocation will assess patients for incident delirium (primary outcome) using the CAM. ${ }^{21}$ Patients who are unable to speak (eg, have a tracheal tube or tracheostomy) will instead be assessed using the confusion assessment method for the intensive care unit (CAM-ICU) instrument. ${ }^{89} 90$ These methods (the CAM and the CAM-ICU) have been shown to be reliable and to have good agreement with the DSM-IV criteria for delirium. ${ }^{90-92}$ In addition to the CAM or the CAM-ICU, an independent trained clinical researcher, blinded to the CAM results, will conduct structured chart reviews to detect episodes of delirium. A combined approach (CAM interview or CAM-ICU plus chart review) increases the sensitivity and retains specificity in detecting incident delirium. ${ }^{93} 94$ Therefore, either a positive clinical delirium assessment (CAM or CAM-ICU) or a positive assessment for delirium based on a validated, structured chart review ${ }^{93}$ will be diagnostic of incident delirium in the ENGAGES trial. The use of the structured chart review will improve sensitivity and contribute to the pragmatic aspects of this trial, since it will abstract incident delirium from a routinely available source. The trial staff will undergo training on the chart review methodology by a skilled chart reviewer under the supervision of Drs Inouye and Schmitt.

The CAM assessment that will be used in the ENGAGES study was developed by Inouye et al. ${ }^{21}$ All ENGAGES CAM assessments will be performed by study team members who have undergone a rigorous training process. Several members of the research team participated in a full-day CAM training programme led by Dr Inouye, the original creator of the instrument. ${ }^{95}$ Those who attended this initial training will oversee the training of other team members. All trainees must demonstrate competence at conducting the structured interviews and in correctly scoring subjects. Trainees must first conduct at least two satisfactory CAM assessments in patients not enrolled in the ENGAGES study in the presence of a trained team member. To establish competency in scoring the CAM, trainees will observe CAM interviews conducted by trained team members and will score the CAM independently. The trainee must agree with the trainer on the presence or absence of all 12 cognitive features assessed by the CAM on a minimum of two delirious and two non-delirious patients. After meeting the stipulations of training, the newly trained team member will conduct their first interview of a patient enrolled into the ENGAGES trial in the presence of a previously trained team member. Independent of the training process, all ENGAGES team members who are participating in CAM assessments must view and rate nine videos of standard interviews of actors depicting delirious and non-delirious patients. This process will help to demonstrate the success of the training process and the extent to which researchers reproducibly score the CAM. To establish the reliability of delirium assessments in the clinical setting, trained members of the research team will separately assess 30 patients not enrolled in the ENGAGES study at similar time points (eg, within 2 hours of each other). These assessments will determine the test-retest reliability of delirium assessments by the research team. This approach will provide $>90 \%$ power to demonstrate a $\kappa$ statistic of $>0.6$, representing substantial or greater agreement between raters. ${ }^{96}$

A structured process will be implemented to assess and ensure the quality of the delirium assessments. Every delirium assessment will be reviewed within 3 days with a fellow member of the research team to assess internal consistency of scoring and completeness. On a weekly basis, investigators at Washington University will review all the delirium assessments, will address methodological inconsistencies and will attempt to resolve controversies. Monthly, there will be a teleconference including investigators from Hebrew SeniorLife/ Harvard University and from Washington University to review challenging delirium assessments and to ensure that the rigour of assessments remains appropriate. During these conferences, the need for focused and comprehensive refresher training in delirium assessment ${ }^{95}$ will be determined.

If participants agree, these assessments may be videotaped for training and education of the research team. The videotapes will include the patients' face.

\section{Health-related quality of life}

As part of the ongoing SATISFY-SOS study, patient selfreported health-related quality of life information will be assessed through the Veteran's RAND 12-item Health Survey at baseline (preoperatively) and during follow-up (30-day and 1-year) (aims 2 and 3 in figure 2). The VR-12 was derived from the Veterans RAND 36 Item Health Survey (VR-36) and contains 12 items relating to quality of life, including physical and mental health, as 
well as specific questions about functional status. Physical and Mental Health Summary Scores will be calculated. The VR-12 has been validated and is widely applied as a metric for tracking health-related quality of life in the USA. ${ }^{97}$

\section{Falls}

In the baseline (preoperative) questionnaire for the SATISFY-SOS study, patients are asked to indicate how many times they fell during the past 6 months and injuries from falls are ascertained (aims 2 and 3 in figure 2). Patients who fell at least one time during the past 6 months will be classified as having a previous history of falls. On the 30-day and 1-year follow-up postoperative questionnaires, patients are asked to indicate whether they have experienced a fall. On the basis of this information, we will define two outcome measures: falls within 30-days and falls within 1-year of surgery. Data on number of falls and injurious falls will also be collected. The wording for the falls questions used in SATISFY-SOS is based on the definition proposed by the Prevention of Falls Network Europe (ProFaNE); the calculation for severity of fall is based on a standard algorithm. ${ }^{98-100}$

\section{Prespecified additional analyses and substudies}

The primary aim of the ENGAGES study is to determine whether an EEG-guided anaesthetic protocol can decrease postoperative delirium and its associated downstream negative sequelae (eg, decrement in quality of life, falls). However, it is important that large, randomised trials collect and report data on multiple clinically relevant outcomes to maximise scientific yield and efficiency. The ENGAGES trial will have the potential to contribute new information on diagnosis of postoperative delirium, risk factors for postoperative delirium and negative outcomes following incident postoperative delirium. The ENGAGES trial will be conducted using the infrastructure of the ongoing SATISFY-SOS study, which is systematically collecting detailed information on surgical patients' characteristics, and is tracking their health and well-being up to 5 years postoperatively. The effect of anaesthetic depth on postoperative morbidity and mortality is currently being explored in the 6500 patient Balanced Anaesthesia Trial. $^{101} 102$ Information provided by the ENGAGES study will add to the growing body of evidence regarding the hypothesised effects of anaesthetic depth on surgical outcomes. Prespecified substudies for the ENGAGES trial are elaborated in an addendum at the end of this protocol.

\section{Patient-centered approach}

Several aspects of the ENGAGES study are patient centred in their conception. There is a community liaison group that has been actively involved in the design and in the conduct of the study. Important outcomes in the study are based on patient-reported outcomes measures. Both patients and their families are provided with educational material on delirium, its risk factors and its sequelae. The study also includes patient self-assessment for delirium and implementation of the validated FAM-CAM ${ }^{103} 104$ instrument in hospital and after hospital discharge. These will be included in prespecified substudies of the ENGAGES trial (see addendum).

\section{Statistical analyses}

Effectiveness of EEG-guided anaesthesia protocol in reducing incident postoperative delirium compared with usual anaesthetic care

We will follow the intention-to-treat principle for all analyses (aim 1 in figure 2). The primary end point of our analysis is the incidence of postoperative delirium as defined previously. The incidence of delirium will be compared between groups using a $\chi^{2}$ test, and the difference in delirium incidence with $95 \%$ CIs will be calculated. Prespecified exploratory subgroup analyses: we will test for effect modification by known baseline delirium risk factors (ie, age $(<70$ and $\geq 70)$, sex, history of falls and type of surgery (cardiac vs non-cardiac)).

\section{Effectiveness of EEG-guided anaesthesia protocol in improving patient-reported outcomes of health-related quality of life and preventing postoperative falls Health-related quality of life}

Random effects regression models based on PROC MIXED in SAS will be used to compare Physical Summary Score change over 12 months between the intervention and usual care group by introducing terms for time, indicator variables for treatment group and timextreatment group interactions with the latter set of regression coefficients of primary interest (aim 2 in figure 2). The model will be controlled for baseline health-related quality of life. Potential confounding factors including age, gender and relevant comorbidities will be evaluated and controlled for in the model. We will use the same analysis approach to evaluate the Mental Summary Scores.

The analysis is primarily based on the following model:

$$
\mathrm{Y}_{\mathrm{it}}=\alpha+\beta_{1} \mathrm{t}+\beta_{2 \mathrm{~A}} \mathrm{X}_{\mathrm{i} 1}+\beta_{3 \mathrm{~A}} \mathrm{X}_{\mathrm{i} 1} \mathrm{t}+\beta_{4} \mathrm{t} \mathrm{Y}_{\mathrm{i} 0}+\mathrm{e}_{\mathrm{it}}
$$

where $Y_{i t}=Q O L$ for subject $i$ at time $t$, where $t=0$ for baseline and $\mathrm{t}=1$ for 12 months. $\mathrm{X}_{\mathrm{i} 1}=1$ if ith subject is in the intervention group, $=0$ otherwise. $e_{i t} \sim N\left(0, \sigma^{2}\right) \beta_{3 A}$ is the mean difference in Physical Summary Score change between the intervention and usual care groups. The coefficient $\beta_{2 \mathrm{~A}}$ allows for mean differences in Physical Summary Scores between groups at baseline. The coefficient $\beta_{4}$ allows the change in Physical Summary Score to depend on initial level.

\section{Postoperative falls}

A $\chi^{2}$ test will be used to compare the incidence of falls at 12 months postoperatively between patients in the EEG-guided and in the usual care groups. 
Explore whether a multicomponent safety intervention is associated with improved patient-reported health-related quality of life and decreased incidence of postoperative falls We will design a prospective matched cohort study using the ENGAGES participants (received multicomponent safety intervention) and SATISFY-SOS patients who are not enrolled in the ENGAGES study (did not receive multicomponent safety intervention) (aim 3 in figure 2). Participants in the ENGAGES study will be matched with reference individuals according to preoperative characteristics. Reference individuals will be identified through the ongoing SATISFY-SOS cohort and will be matched by age $( \pm 1$ year $)$, American Society of Anesthesiologists' physical status (1-4), type of surgery (cardiac vs non-cardiac), date of planned surgery $( \pm 1$ year) and history of falls (yes or no). Health-related quality of life and fall incidence will be compared between these matched cohorts at $\sim 1$ month and 1 year postoperatively.

All statistical analyses will be performed using SAS, V.9.4 (SAS Institute, Cary, North Carolina, USA). All tests will be two sided and by arbitrary convention ${ }^{105}$ will be considered statistically significant at a $\mathrm{p}<0.05$, and all results will be presented with estimates and 95\% CIs. However, on the basis of the uncertain prior probability (plausibility) of the alternative hypothesis ${ }^{106}$ (ie, EEG guidance of anaesthesia decreases postoperative delirium) and concerns raised about lack of reproducibility in science, ${ }^{107} 108$ a statistically significant result with a $\mathrm{p}$ value just $<0.05$ should be considered as preliminary, and future studies should be conducted for corroboration. A more stringent $\mathrm{p}$ value $(\mathrm{eg}, \mathrm{p}<0.005)$ would be required to conclude that subsequent studies would be very likely to reproduce these results with a $p$ value $<0.05 .{ }^{109}$ Apart from statistical significance, the ultimate decision regarding the routine implementation of EEG guidance of general anaesthesia in preventing postoperative delirium will also depend on the estimated effect size of this intervention.

\section{Sample size calculations}

All sample size calculations have been performed using SAS PROC POWER.

\section{Decrease in delirium}

Our sample size calculations are based on the anticipated delirium incidence and effect size for our primary end point analysis. On the basis of results of a previously published meta-analysis of four studies investigating the use of BIS-guided anaesthetic administration, we conservatively assume an incidence of postoperative delirium in the routine anaesthesia care group of $25 \%{ }^{29}$ We performed a sample size sensitivity analysis and calculated different scenarios with different values for the delirium incidence in the intervention arm and corresponding power $(80 \%, 90 \%$ and $95 \%)$. With a two-sided $\alpha<5 \%$ and 1232 patients (616 per arm), the trial will have $>95 \%$ power to detect an absolute decrease in delirium incidence $\geq 9 \%$, $>90 \%$ power to detect a decrease in delirium incidence $\geq 8 \%$ and $>80 \%$ power to detect a decrease in delirium incidence $\geq 7 \%$. With a $7 \%$ decrease in delirium incidence, the $95 \%$ CI would be $\sim 3-12 \%$. Even a $3 \%$ decrease in delirium incidence would be clinically important, suggesting that delirium would be prevented in one out of every 33 at risk patients who received EEG guidance of general anaesthesia.

\section{Health-related quality of life}

The overall sample size of our study is defined through estimations of the primary outcome (delirium). For secondary analyses, we assume that $\sim 80 \%$ of our trial population ( 1000 participants) will have completed the trial and the 1-year follow-up survey. With this sample size of 1000 , we can detect a difference of 0.5 points (SD of 2.5) in the mean change of Physical Health Score from baseline to 1 year between the intervention and usual care group with a power of $>80 \%$ and a two-sided $\alpha$ level of $\mathrm{p}<0.05$.

\section{Postoperative falls}

This calculation is similarly based on the assumption that $\sim 80 \%$ of our trial population ( 1000 participants) will have completed the trial and the 1-year follow-up survey. On the basis of a study that showed a preoperative fall prevalence of $33 \%$ over 6 months preoperatively in a similar patient population to the ENGAGES trial, we will conservatively assume an incidence of postoperative falls at 1-year in the routine anaesthesia care group of $40 \% .^{110}$ With a sample size of 1000 , we will have $>80 \%$ power to detect an absolute risk reduction of $12 \%$ between the EEG-guided and the usual care group at a two-sided $\alpha$ level of $\mathrm{p}<0.05$.

\section{Analysis of pragmatic elements of the ENGAGES study}

According to seven of nine criteria elaborated in the pragmatic-explanatory continuum indicator summary (PRECIS-2) tool, ${ }^{111}$ the ENGAGES trial is designed predominantly as a pragmatic rather than as an explanatory study (see figure 6). (1) Regarding eligibility criteria, all surgical patients older than 60 undergoing major surgical procedures are eligible for the ENGAGES study, regardless of other known risk factors for delirium. As such, this is broadly representative of a substantial population of older surgical patients. However, the results would not apply to younger patients or to older patients undergoing non-invasive surgical procedures. (2) Patients are recruited in usual clinical settings with slightly more effort made over and above what would be used in the usual care setting to engage with patients. (3) The trial is being conducted in usual care settings, predominantly in the operating rooms and in hospital wards. (4) Any anaesthesia practitioner, regardless of their background or expertise in EEG monitoring, can apply the EEG-guided protocol. However, during the first phase of the study, there will be structured 


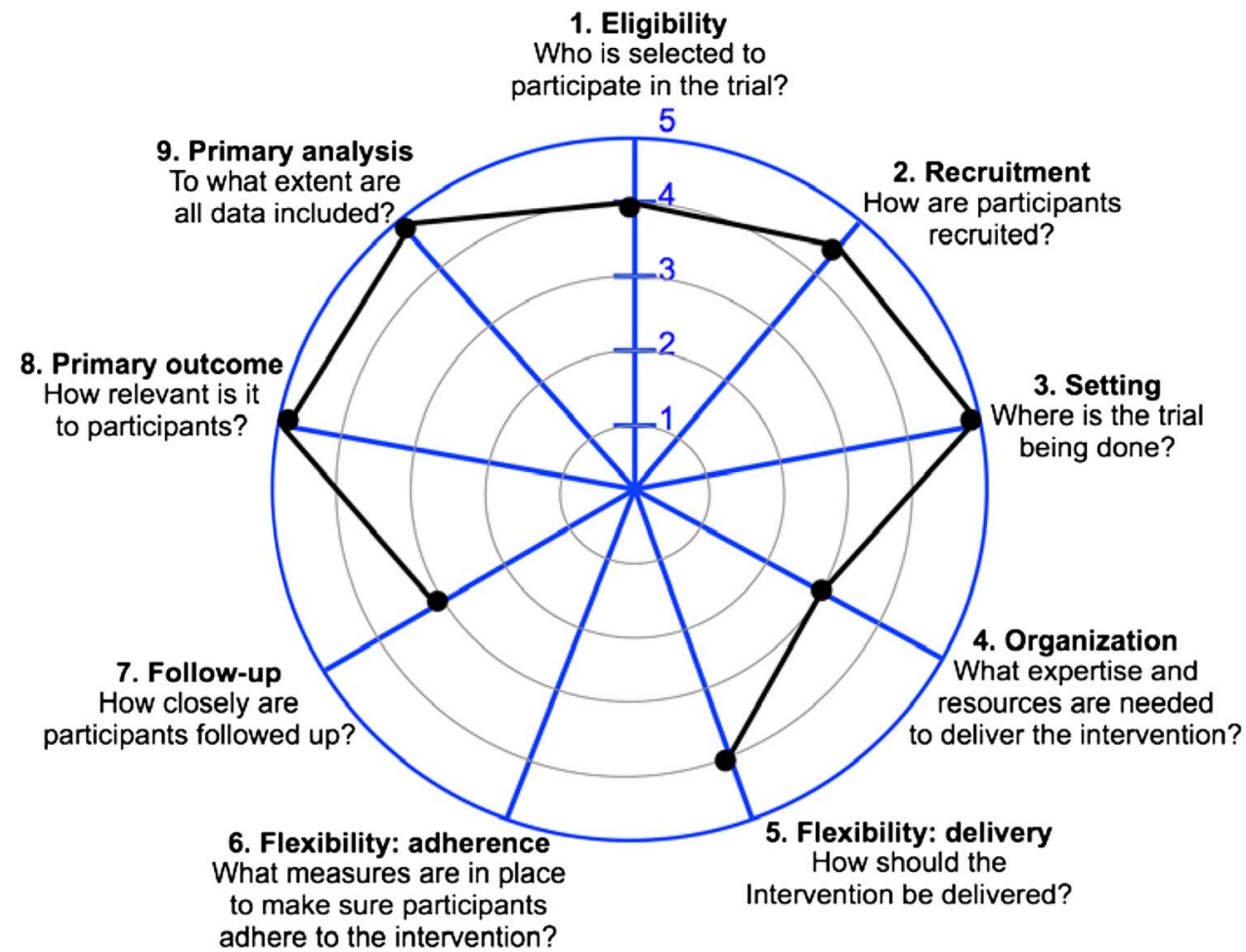

Figure 6 The design elements of the ENGAGES trial that tend to be pragmatic (markers placed towards the periphery) and elements that tend to be explanatory (markers placed towards the centre). ${ }^{111} 113$ This figure was generated from a median determination for each criterion (using a 1 to 5 ordinal scale from explanatory to pragmatic) from 18 independent raters on the study team. Aside from the intensity of patient follow-up and the expertise needed to deliver the EEG-guided protocol, the ENGAGES study fulfils the criteria for a pragmatic clinical trial. ${ }^{111} 112$

education of clinicians to increase their familiarity and comfort with EEG guidance of anaesthesia. We therefore anticipate that practitioner familiarity with EEG will increase over the course of the study, although only basic knowledge regarding EEG analysis will be needed. Similarly, any anaesthesia practitioner, regardless of their background or expertise, can apply the control (comparison) protocol. (5) Instructions on how to apply the EEG-guided protocol are flexible, offering practitioners discretion in deciding how to formulate and apply it. Although clinicians carrying out the EEG-guided protocol will use their own discretion in managing anaesthesia, there is an expectation that less anaesthesia will be administered in the EEG-guided arm and that the cumulative duration of EEG suppression will be less in the EEG-guided arm. During the first phase of the study, clinician adherence to the EEG-guided protocol will be evaluated and will partially inform the value of proceeding with the second phase of the study. Similar to the intervention arm, when patients are randomised to the control arm, anaesthesia clinicians will have leeway to pursue their usual practice with minimal restrictions. There are some limitations in relation to the anaesthetic technique (eg, based on potent volatile anaesthetic); however, these are consistent with current practice at our institution and more broadly. (6) For the primary intervention of the
ENGAGES trial, participants will be anaesthetised and will have no ability to impact adherence to the intervention. Therefore, this domain was left blank in the PRECIS-2 determination, as recommended. ${ }^{111}$ Patients enrolled to the ENGAGES trial will be followed with more frequent visits and more extensive data collection than would occur in routine practice. (8) Incident delirium, the primary outcome of the study, is an objectively measured, clinically meaningful outcome to the study participants. The outcome can be assessed under usual conditions and typically does not rely on central adjudication. However, special training in rigorous delirium assessment is required. It is important to note that the abstraction of information about delirium from the medical records bolsters the pragmatic aspects of the trial, since this is an information source that is readily available at any hospital. (9) The analysis of the results will include all patients regardless of clinician compliance with the EEG-guided protocol (ie, it will be an 'intention-to-treat' analysis). The analysis will attempt to determine whether or not the EEG-guided protocol prevents postoperative delirium under the usual conditions, with all the noise inherent therein. Although in most respects the ENGAGES trial was judged to be pragmatic, this appraisal might have been biased as it was conducted by investigators associated with the study. ${ }^{112}$ 


\section{Strengths and limitations}

The ENGAGES study has important strengths. It is largely a pragmatic randomised clinical trial conducted in a high volume, real world clinical setting that incorporates an easy-to-implement intervention and examines an outcome that is of tremendous importance to patients, healthcare providers and society. The ENGAGES study can be conducted efficiently as many components of the proposed study are incorporated into existing infrastructures and processes at Washington University: (1) enrolment will be integrated into the flow of the CPAP; (2) the conduct of the study will largely be by anaesthesiologists and certified registered nurse anaesthetists in the course of their routine clinical work and (3) most of the follow-up data will be obtained from SATISFY-SOS, an ongoing registry study. Randomisation can be implemented easily at the point of patient care, as the anaesthesia protocols do not require any lead-in time or advanced preparation. The study will enrol older patients, who are recognised to be vulnerable and understudied in clinical research. This targeted population is especially important to understand and would stand to benefit significantly from reductions in postoperative delirium and related outcomes. The secondary outcomes of the study include patient-reported health-related quality of life, which is extremely relevant to patients. The study is also designed to detect postoperative falls and their potential prevention. The trial will exploit the extensive SATISFY-SOS prospective patient registry and our highly evolved perioperative electronic medical record. Most of the data collected for the trial will use existing infrastructure, and additional data will be entered using the REDCap resource that integrates well with our other data repositories. The feasibility of the trial is enhanced by participation of a multidisciplinary team of investigators that has now established a track record of collaboration and completion of major clinical trials. As the intervention is inexpensive and straightforward, if the results of the study show compelling effectiveness, it will be logistically simple to implement and sustain the EEG-guided anaesthesia protocol at our institution and disseminate it nationally in the USA.

The following limitations should be considered. A single clinical trial should seldom be regarded as definitive. As there is no clear estimate for the prior probability that EEG guidance of anaesthesia prevents postoperative delirium, if the results of this trial do suggest that EEG guidance of anaesthesia might decrease delirium at the arbitrary statistical threshold of $\mathrm{p}<0.05$, it will be necessary to replicate this finding in future studies. On the other hand, even if the study finds a non-significant (at the arbitrary threshold of $\mathrm{p}>0.05)$ decrease in delirium in the EEG-guided group, it is likely that follow-up studies will be warranted to clarify whether or not there is a clinically meaningful reduction in delirium with EEG guidance of anaesthesia, and whether there are specific patient populations that might especially benefit from this intervention. If EEG guidance of anaesthesia can prevent postoperative delirium, demonstrating its effectiveness will depend on clinicians' adherence to the protocol. However, the inability to blind clinicians to the trial allocation group is a potential source of bias and confounding. We are attempting to confirm that clinicians do alter anaesthetic management based on the intervention during the pilot phase of the study. The inclusion of patients in a clinical trial focused on the prevention of delirium, and the provision of practical educational information to patients and family members could decrease the incidence of postoperative delirium. Furthermore, if the multicomponent intervention is successful in preventing falls and in improving quality of life, this could curtail our ability to detect an impact of the EEG-guided anaesthetic protocol on these outcomes. In addition to the pragmatic structured chart review, there are two clinical assessment methods that will be used to diagnose delirium: the CAM-ICU and the CAM. The CAM-ICU is less sensitive than the CAM, but is the only instrument that has been validated for patients who are non-verbal (ie, with a breathing tube or tracheostomy in place). On the basis of our institutional data, the vast majority of patients enrolled in the study will be extubated within the first two postoperative days. Therefore, most patients will have delirium assessments with the CAM, which is the more sensitive and specific instrument. We will also test whether intubation status modifies the result in secondary analysis. The study design includes a 30-day and a 1-year follow-up for patient-reported outcomes, and incomplete follow-up is therefore a potential limitation. On the basis of our previous B-Unaware and BAG-RECALL studies, ${ }^{40}{ }^{41}$ we are confident that we can achieve a 30-day follow-up rate of $>90 \%$. In our SATISFY-SOS cohort, the 1-year follow-up has yielded $\sim 66 \%$ response rate. We have performed sensitivity analysis in our power calculations and have taken into account this potential attrition in our methods. Furthermore, we plan to enhance follow-up by using supplementary phone calls from members of the study team. As delirium is a fluctuating disorder, there is a risk that it can be missed by periodic assessments. We are attempting to mitigate this by assessing patients for delirium during a time of day (afternoon/evening) when delirium occurs more commonly. Furthermore, we are incorporating structured chart review, which has been validated as a complementary approach that increases the detection of delirium. ${ }^{93} 94$

\section{Potential benefits, risks and alternatives Benefits}

If the hypotheses motivating this study are correct, patients who are randomised to receiving EEG guidance of anaesthesia will have a lower chance of experiencing postoperative delirium and possibly also its downstream consequences, including quality of life decrement and injurious falls. All the patients enrolled in this study will 
potentially benefit from the safety interventions intended to decrease the likelihood of postoperative falls.

\section{Risks}

The risks associated with this study are low. There is a rare risk of breach of confidentiality. The main risk attributable to the EEG-guided intervention might be increased risk of intraoperative awareness. This is unlikely as previous studies that have randomised patients to EEG guidance have not found an increased incidence of awareness with EEG-based anaesthetic protocols. ${ }^{40-42}$ However, limitations of the BIS in detecting awareness in the presence of neuromuscular blocking agents have recently been highlighted. ${ }^{114}$ Titration of anaesthesia in the ENGAGES trial is therefore based primarily on the raw EEG waveform and only secondarily on the processed EEG index. Nonetheless, as a potential safety concern regarding the EEG-guided intervention remains that it could increase the incidence of intraoperative awareness, this outcome will be tracked postoperatively with a modified Brice interview ${ }^{115}$ conducted within 48 hours of extubation. In addition, questions regarding intraoperative awareness are also included in the SATISFY-SOS 1-month survey. A data-safety monitoring committee will review adverse events with the PI and, in consultation with the institutional review board, might recommend stoppage of the trial if awareness events appear to be increased in the intervention group. As part of the informed consent process for this study, patients will be informed of the rare risk of awareness. In the unlikely event that serious side effects occur, they will be documented and will be reported to the human research protection office and to the study's data safety monitoring board. Participants will not incur any studyrelated expenses, nor will they be financially compensated for their participation.

\section{Minimization of risks and confidentiality}

Necessary protected health information will only be shared with members of the research team. To help protect confidentiality, research charts will be stored in a locked cabinet inside the locked research office. Electronic data and demographic information will also be kept in a password-protected electronic database stored on the departmental network drive only accessible via password-protected departmental computers. A member of the research team will enter this information. Only code numbers will appear on any data and documents used for evaluation or statistical analyses. Patients may choose not to participate in this study, and there will be no penalty in terms of the care that they receive.

The Division of Biostatistics Informatics Core at Washington University will be used for data processing and management. Washington University belongs to a consortium of institutional partners that work to maintain a software toolset and workflow methodology for electronic collection and management of research and clinical trial data. REDCap (Research Electronic Data Capture) data collection projects rely on a thorough study-specific data dictionary defined in an iterative selfdocumenting process by all members of the research team with planning assistance from the Division of Biostatistics Informatics Core. The iterative development and testing process result in a well-planned data collection strategy for individual studies. REDCap servers are securely housed in an on-site limited access data centre managed by the Division of Biostatistics at Washington University. All web-based information transmission is encrypted. The data are all stored on a private, firewallprotected network. All users are given individual user identifiers and passwords, and their access is restricted on a role-specific basis. REDCap was developed specifically around HIPAA-Security guidelines and is implemented and maintained according to Washington University guidelines. REDCap currently supports $>500$ academic/ non-profit consortium partners on 6 continents and 38800 research end users.

\section{Adverse event reporting and safety monitoring}

The research team will monitor the study for adverse events. All serious adverse events (SAEs) will be reported to the IRB according to IRB stipulations. The monitoring plan for this study is appropriate for the planned pragmatic trial. We have already conducted three large clinical studies including 28 000 patients, half of whom received general anaesthesia with EEG guidance. There were no adverse events attributable to EEG guidance of anaesthesia in these studies; ${ }^{40-42}$ it is unlikely that there will be adverse events attributable to EEG guidance in the ENGAGES study.

The ENGAGES has an appropriate data and safety monitoring plan for a low-risk clinical trial. There is a charter to guide the functions of the DSMB, and the DSMB will produce reports in accordance with NIH guidelines. The DSMB will provide independent oversight of the ENGAGES Clinical Trial and will review general conduct of the trial and study data for participant safety. ${ }^{116}$ The DSMB is comprised of independent, multidisciplinary experts who will make recommendations regarding the continuation, modification or termination of the trial. ${ }^{117}$ The members will have the requisite expertise to examine accumulating data, to protect the integrity of the clinical experiments to which the patients have consented to participate and to assure the regulatory bodies, the public and the NIH that conflicts of interest do not compromise either patient safety or trial integrity. ${ }^{118}$ The DSMB will convene twice annually to review safety events. There will be a provision for early stoppage for safety concerns but not for efficacy or for futility. ${ }^{116}$ Trials that stop early for benefit show implausibly large treatment effects, particularly when the number of events is small. ${ }^{119}$ Truncated trials have been associated with greater effect sizes than trials not stopped early, independent of the presence of statistical stopping rules. ${ }^{120}$ The members of the DSMB shall have 
no direct involvement in the conduct of the ENGAGES study. Neither shall they have financial, proprietary or professional conflicts of interest, which may affect the impartial, independent decision-making responsibilities of the DSMB. ${ }^{116}{ }^{117}$ All DSMB members have signed a Conflict of Interest Certification to confirm no conflict exists. There are five people on the DSMB to optimise performance. ${ }^{121}$ The DSMB will be advisory rather than executive on the basis that it is the ENGAGES study investigators in partnership with the National Institute on Aging who are ultimately responsible for the conduct of the trial (see figure 7$){ }^{121}$

\section{Premature study termination}

Patients in the EEG-guided anaesthetic group will, on average, receive decreased concentrations of inhaled anaesthetic agents during their surgeries. Reduction of anaesthetic administration using simultaneous EEG-based monitoring of anaesthetic depth has been previously described without reports of increased intraoperative awareness. ${ }^{27} 122$ However, it is theoretically plausible that a significantly higher rate of awareness events could occur in a cohort that on average receives lower anaesthetic concentrations. Therefore, we propose comparing the incidence of intraoperative awareness reports in the EEG-guided and usual care groups. We will recommend to the DSMB that this occurs after 600 patients have been enrolled. A one-tailed comparison will be used to compare the incidences of awareness in the groups, and consideration should be given to terminating the study if the EEG-guided cohort has a significantly greater incidence of intraoperative awareness compared with the standard of care group with a $\mathrm{p}$ value $<0.05$. In making recommendations, the DSMB could take into consideration the severity of the awareness experiences, including reports of pain, paralysis and distress. ${ }^{123}$ Apart from intraoperative awareness, it is not currently hypothesised that decreased anaesthetic administration is associated with clinically relevant adverse outcomes (eg, death, myocardial infarction, stroke). It is possible that decreases in anaesthetic administration might be associated with intraoperative patient movement, or with increased intraoperative blood pressure and heart rate. However, these are surrogate measures with unclear clinical relevance, which should not therefore impact a decision to terminate the study early.

We recommend to the DSMB not performing an interim analysis of delirium rates for any consideration of termination. Currently, available data support the possibility that an EEG-guided anaesthetic management to reduce anaesthetic administration might decrease the incidence of postoperative delirium or have no effect on this outcome. ${ }^{26-29}$ Conversely, the possible finding of a higher incidence of delirium in the EEG-guided cohort would conflict with current evidence. When interpreted in the context of existing evidence, the finding of significantly disparate incidences of postoperative delirium in a partially completed ENGAGES trial would not provide a sufficient evidence base to change the standard of practice for anaesthetic guidance of these patients.

\section{Indemnity}

Washington University School of Medicine is responsible for any non-negligent damage incurred as a result of participating in the ENGAGES trial. The indemnity is renewed on an annual basis. Washington University School of Medicine assures that it will continue renewal of the indemnity for the duration of the trial.

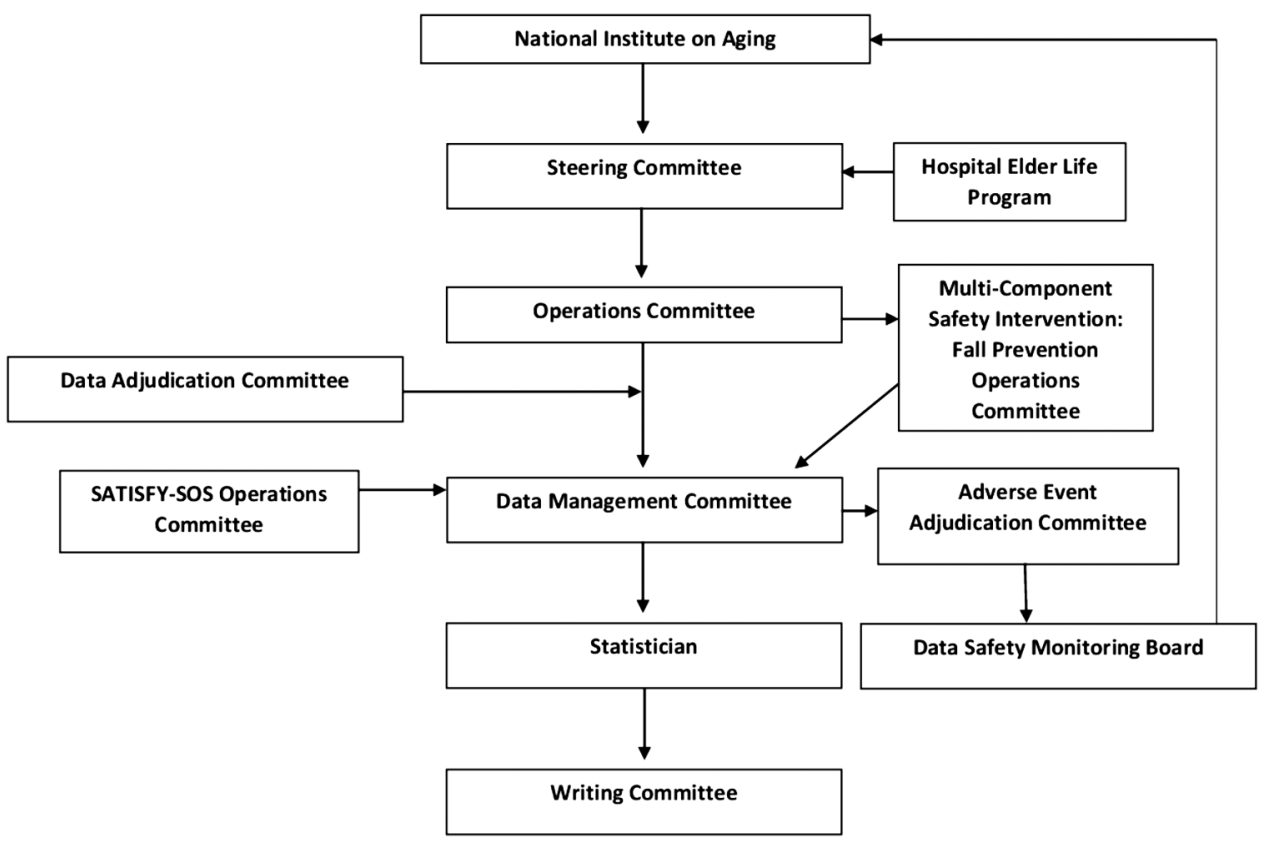

Figure 7 ENGAGES trial organization. 


\section{Ethics and dissemination}

The trial steering committee will be responsible for all major decisions regarding changes to the protocol. The committee will communicate these changes to the IRB and appropriate parties. The final trial data set is the property of the investigative team and shall not be shared without permission from the principal investigator. Data will be shared with the National Institute on Aging. Dissemination plans include presentations at local, national and international scientific conferences. Every effort will be made to publish results of the ENGAGES trial in a peer-reviewed journal. Dissemination of results to study participants and their family members will be available upon request. Updates and results of the study will be available to the public at clinicaltrials.gov.

\section{ADDENDUM}

\section{Prespecified substudies}

A. Duration and severity of delirium: In addition to the incidence of delirium (the primary outcome of the ENGAGES study), other outcomes of interest will be the duration of delirium and the severity of delirium, both of which have been shown to have prognostic importance. ${ }^{124-128}$ The severity of delirium will be scored using the CAM-Severity (CAM-S) metric, which has specifically been shown to be strongly associated with clinically relevant outcomes. ${ }^{128}$ Delirium will also be assessed postoperatively on the day of surgery, when patients are sufficiently awake (RASS >-4).

B. Agreement among the FAM-CAM, researchers' delirium assessments and patient perceptions: The ENGAGES study is a patient-centred study. As such, the active involvement of patients and their families is an important component. The family confusion assessment method (FAM-CAM) instrument has previously been shown to have good agreement with the CAM and with DSM-IV diagnostic criteria in patients with cognitive impairment and in hospitalised patients. ${ }^{103} 104$ The utility of the FAM-CAM has not been established in postoperative patients; however, it has been successfully implemented in the postoperative setting in the ongoing PODCAST clinical trial. ${ }^{129}$ Patients will also complete a delirium selfassessment questionnaire (see online supplementary appendix). The FAM-CAM assessments and the patients' self-assessments will be compared with the researchers' delirium assessments.

C. Duration or recurrence of delirium after hospital discharge as measured by the FAM-CAM and patient perceptions: Little is currently known about either duration of delirium or recurrence of delirium after hospital discharge in postoperative patients. The FAM-CAM and patient self-reports will be used to assess these outcomes.

D. Clinically relevant outcomes associated with delirium: Delirium incidence, duration and severity have all been shown to be associated with other (downstream) clinically relevant outcomes, including mortality, length of ICU stay, length of hospital stay, falls, cognitive decline and functional decline. In the ENGAGES study, these associations will be explored. The data on downstream outcomes will be obtained from hospital records or from patient-reported outcome measures that are collected as part of the ongoing SATISFY-SOS study.

E. Comparison of patient-reported and observational pain scores: It is likely that patients with delirium are less able to convey verbally the extent to which they are in pain. ${ }^{130}$ Given that postoperative delirium is common and may relate to uncontrolled pain, this has important implications for the assessment and treatment of postoperative pain. We plan to compare patientreported and behavioral pain assessments in nondelirious and delirious patients ${ }^{130}$ (see online supplementary appendices for pain assessment instruments)

F. Postoperative actigraphy and EEG: Postoperative disturbances in sleep and EEG abnormalities have previously been associated with postoperative delirium. ${ }^{131-134}$ EEG data will be collected from some patients at around the time of delirium assessments. Patients might also wear actigraphy watches to help distinguish episodes of sleep from wakefulness in the postoperative period. ${ }^{135}$

G. Relationship between clinical CAM-ICU and rigorous delirium assessments: Routine clinical (ie, conducted by ICU nursing staff) delirium assessments in the intensive care units (conducted with the CAM-ICU) will be collected when these are available. Comparison will be made between these routine clinical assessments and the assessments made by the research team.

H. Association between delirium and patient outcomes: The ENGAGES study will evaluate the association between postoperative delirium and patient-reported outcome metrics, including quality of life and falls, up to 1 year postoperatively.

I. Postoperative outcomes hypothesised to be associated with anaesthetic depth: It is likely that patients randomised to the EEG-guided protocol will be exposed to lower concentrations of anaesthetic agents and on average will not be as deeply anaesthetised. There is an ongoing randomised, clinical trial investigating the effects of depth of anaesthesia on a range of outcomes, ${ }^{101} 102$ including death, myocardial infarction, cardiac arrest, pulmonary embolus, stroke, surgical site infection, ICU length of stay, hospital length of stay, intraoperative awareness, persistent pain and cancer recurrence. Many of these outcomes are tracked with the SATISFY-SOS study and will therefore be reported for patients enrolled in the ENGAGES study.

J. Delirium prediction models: It is important to improve our understanding of factors that are associated with an increased incidence of postoperative delirium or perhaps may even mediate an elevated 
risk for postoperative delirium. Previous studies have explored risk factors, usually using logistic regression models. $^{29}{ }^{136-147}$ In a previous study, we used a Bayesian exploratory approach with a stochastic search variable selection method. ${ }^{29}$ The ENGAGES study will rigorously assess a large number of surgical patients for postoperative delirium, and it will therefore lend itself to further exploration, refinement of risk models and hypothesis generation. On the basis of the results from previous studies, we will include specific variables in our analyses. Patient age, demographic, lifestyle and comorbidity information will be assessed at baseline through a standardised interview in the CPAP clinic. Data on previously described risk factors for postoperative delirium will be acquired including history of postoperative delirium, modified Charlson Comorbidity Index, American Society of Anesthesiologists' Physical Status, functional status, level of education, olfaction, baseline cognition, depression (using the PHQ-9 questionnaire), indices of frailty, obstructive sleep apnoea, baseline haematocrit, baseline sodium and creatinine, preoperative psychoactive medications (eg, opioids, benzodiazepines, sedatives, clonidine), alcohol use, dosages of perioperative medications (eg, hypnotic anaesthetics, opioids, benzodiazepines, dexmedetomidine), intraoperative hemodynamic parameters, other physiological parameters, processed EEG indices (eg, BIS, burst suppression), vasoactive medications, perioperative blood transfusions, postoperative mechanical ventilation, postoperative pain (using Visual Analogue Scale and Behavioural Pain Scale), postoperative sleep deprivation, postoperative medical complications, postoperative shock, postoperative anaemia (haematocrit $<30 \%$ ), postoperative hypoalbuminemia (albumin $<3 \mathrm{~g} / \mathrm{dL}$ ), postoperative temperature and postoperative sodium concentration. ${ }^{29} 79$ 136-147

\section{Author affiliations}

${ }^{1}$ Department of Anesthesiology, Washington University School of Medicine, St. Louis, Missouri, USA

${ }^{2}$ Division of Public Health Sciences, Department of Surgery, Washington University School of Medicine, St. Louis, Missouri, USA

${ }^{3}$ Department of Psychiatry, Washington University School of Medicine, St. Louis, Missouri, USA

${ }^{4}$ Department of Occupational Therapy, Washington University Institute for Public Health, School of Medicine, St. Louis, Missouri, USA

${ }^{5}$ Department of Neurology, Washington University Institute for Public Health, School of Medicine, St. Louis, Missouri, USA

${ }^{6}$ Department of Mathematics, Biostatistics Division, Washington University in St. Louis, St. Louis, Missouri, USA

${ }^{7}$ Department of Medicine, Beth Israel Deaconess Medical Center, Harvard Medical School, Boston, Massachusetts, USA

${ }^{8}$ Institute for Aging Research, Hebrew SeniorLife, Boston, Massachusetts, USA

${ }^{9}$ Department of Surgery, Cardiothoracic Division, Washington University School of Medicine, St. Louis, Missouri, USA

${ }^{10}$ Department of Anesthesia \& Perioperative Care, University of California San Francisco, San Francisco, California, USA

${ }^{11}$ Department of Anesthesia, University of Manitoba/Winnipeg Regional Health Authority Anesthesia Program, Winnipeg, Manitoba, Canada
Collaborators The authors thank Will Godfrey, Furqaan Sadiq, Nicole Shin, Rachel Steinhorn, Laura Veremakis, Maxim Wolfson, Guoquan Xu, Melissa Milbrandt, Phil Vlisides, George Mashour, Stacey Dahm, Emily Somerville, Aamil Patel, Jamila Burton, Jennifer Tappenden, Catherine Tedeschi, Sharon Jiang, Jenny Zhao Cheng, Victoria Cui and Yulong Chen. The ENGAGES study is being conducted in collaboration with complementary trials at the University of California, San Francisco (UCSF) (NCT01983384) and the University of Manitoba in Winnipeg (NCT02692300). Some of the outcomes will be analysed considering data from some or all of these studies, as appropriate. In terms of the practicality of disseminating the EEG-guided protocol in North America and beyond, it will be important to demonstrate the feasibility and impact of the protocol in multiple sites.

Contributors Authorship for this study will be given to key personnel involved in study design, recruitment, data collection and data analysis. There are no publication restrictions, and no professional writers will be involved in the generation of the manuscript. MSA, DE, KEE, BAF, TJG, RLH, SKI, EJ, EJL, JML, NL, SJM, BJP, EMS, SS, TWS, BT, TSW and ACW are responsible for conceptualising study design. SLM managed patient safety protocol and IRB compliance. HRM, AMM, MRM, MRMur and RTU were responsible for recruitment, enrolment, data collection and editing the protocol. MSA is responsible for drafting the protocol. All authors including MSA, DAE, KEE, BAF, TJG, RLH, SKI, EJ, EL, EJL, NL, HRM, SLM, SJM, AMM, MRM, MRMur, BJP, EMS, SS, TWS, BT, RTU, TSW and ACW have critically revised the ENGAGES protocol and approved the final version. All authors agree to be accountable for the accuracy and integrity of all aspects of the ENGAGES trial.

Funding Funding for the ENGAGES trial was through a UH2/UH3 mechanism grant awarded by the National Institute on Aging (award reference number 1UH2AG050312-01). Funding for the SATISFY-SOS study was from a grant awarded by the Barnes-Jewish Hospital Foundation (award reference number 7937-77) and support provided by the Department of Anesthesiology at Washington University. In addition, resources for this study and the time of SKI and DS were covered in part by grants (nos. P01AG031720, K07AG041835 and R01AG044518).

Competing interests None declared.

Ethics approval Washington University Human Research Protection Office.

Provenance and peer review Not commissioned; externally peer reviewed.

Data sharing statement The submitted manuscript is a protocol. Data collection is ongoing.

Open Access This is an Open Access article distributed in accordance with the Creative Commons Attribution Non Commercial (CC BY-NC 4.0) license, which permits others to distribute, remix, adapt, build upon this work noncommercially, and license their derivative works on different terms, provided the original work is properly cited and the use is non-commercial. See: http:// creativecommons.org/licenses/by-nc/4.0/

\section{REFERENCES}

1. Projected Future Growth of the Older Population. http://www.aoa. gov/AoARoot/Aging_Statistics/future_growth/future_growth.aspx age (accessed 18 Jan 2014).

2. Inpatient Surgery. http://www.cdc.gov/nchs/fastats/insurg.htm (accessed 18 Jan 2014)

3. Whitlock EL, Vannucci A, Avidan MS. Postoperative delirium. Minerva Anestesiol 2011;77:448-56.

4. European Delirium Association; American Delirium Society. The DSM-5 criteria, level of arousal and delirium diagnosis: inclusiveness is safer. BMC Med 2014;12:141.

5. Inouye SK. Delirium in older persons. $N$ Engl J Med 2006;354:1157-65.

6. Leslie DL, Marcantonio ER, Zhang Y, et al. One-year health care costs associated with delirium in the elderly population. Arch Intern Med 2008;168:27-32.

7. Gottesman RF, Grega MA, Bailey MM, et al. Delirium after coronary artery bypass graft surgery and late mortality. Ann Neurol 2010;67:338-44.

8. Koster S, Hensens AG, Schuurmans MJ, et al. Consequences of delirium after cardiac operations. Ann Thorac Surg 2012;93:705-11. 
9. Kat MG, Vreeswijk R, de Jonghe JF, et al. Long-term cognitive outcome of delirium in elderly hip surgery patients. A prospective matched controlled study over two and a half years. Dement Geriatr Cogn Disord 2008;26:1-8.

10. Bickel H, Gradinger R, Kochs E, et al. High risk of cognitive and functional decline after postoperative delirium. A three-year prospective study. Dement Geriatr Cogn Disord 2008;26:26-31.

11. Toye C, Matthews A, Hill A, et al. Experiences, understandings and support needs of family carers of older patients with delirium: a descriptive mixed methods study in a hospital delirium unit. Int $J$ Older People Nurs 2014;9:200-8.

12. Inouye SK, Rushing JT, Foreman MD, et al. Does delirium contribute to poor hospital outcomes? A three-site epidemiologic study. J Gen Intern Med 1998;13:234-42.

13. Marcantonio ER, Flacker JM, Michaels $\mathrm{M}$, et al. Delirium is independently associated with poor functional recovery after hip fracture. J Am Geriatr Soc 2000;48:618-24.

14. Zhang Z, Pan L, Ni H. Impact of delirium on clinical outcome in critically ill patients: a meta-analysis. Gen Hosp Psychiatry 2013;35:105-11.

15. O'Keeffe S, Lavan J. The prognostic significance of delirium in older hospital patients. J Am Geriatr Soc 1997;45:174-8.

16. Witlox J, Eurelings LS, de Jonghe JF, et al. Delirium in elderly patients and the risk of postdischarge mortality, institutionalization, and dementia: a meta-analysis. JAMA 2010;304:443-51.

17. Cole MG, Ciampi A, Belzile E, et al. Persistent delirium in older hospital patients: a systematic review of frequency and prognosis. Age Ageing 2009;38:19-26.

18. Saczynski JS, Marcantonio ER, Quach L, et al. Cognitive trajectories after postoperative delirium. $N$ Engl J Med 2012;367:30-9.

19. Wacker $\mathrm{P}$, Nunes $\mathrm{PV}$, Cabrita $\mathrm{H}$, et al. Post-operative delirium is associated with poor cognitive outcome and dementia. Dement Geriatr Cogn Disord 2006;21:221-7.

20. Inouye SK, Westendorp RG, Saczynski JS. Delirium in elderly people. Lancet 2014;383:911-22.

21. Inouye SK, van Dyck $\mathrm{CH}$, Alessi CA, et al. Clarifying confusion: the confusion assessment method. A new method for detection of delirium. Ann Intern Med 1990;113:941-8.

22. Inouye SK, Bogardus ST Jr, Charpentier PA, et al. A multicomponent intervention to prevent delirium in hospitalized older patients. N Engl J Med 1999;340:669-76.

23. Inouye SK, Baker DI, Fugal P, et al. Dissemination of the Hospital Elder Life Program: implementation, adaptation, and successes. J Am Geriatr Soc 2006;54:1492-9.

24. Fox MT, Sidani S, Persaud M, et al. Acute care for elders components of acute geriatric unit care: systematic descriptive review. J Am Geriatr Soc 2013;61:939-46.

25. Fox MT, Persaud M, Maimets I, et al. Effectiveness of acute geriatric unit care using acute care for elders components: a systematic review and meta-analysis. J Am Geriatr Soc 2012;60:2237-45.

26. Sieber FE, Zakriya KJ, Gottschalk A, et al. Sedation depth during spinal anesthesia and the development of postoperative delirium in elderly patients undergoing hip fracture repair. Mayo Clin Proc 2010;85:18-26.

27. Chan MT, Cheng BC, Lee TM, et al. BIS-guided anesthesia decreases postoperative delirium and cognitive decline. J Neurosurg Anesthesiol 2013;25:33-42.

28. Radtke FM, Franck M, Lendner J, et al. Monitoring depth of anaesthesia in a randomized trial decreases the rate of postoperative delirium but not postoperative cognitive dysfunction. Br J Anaesth 2013;110(Suppl 1):i98-105.

29. Whitlock EL, Torres BA, Lin N, et al. Postoperative delirium in a substudy of cardiothoracic surgical patients in the BAG-RECALL clinical trial. Anesth Analg 2014;118:809-17.

30. Rampil IJ. A primer for EEG signal processing in anesthesia. Anesthesiology 1998;89:980-1002.

31. Kertai MD, Pal N, Palanca BJ, et al Association of perioperative risk factors and cumulative duration of low bispectral index with intermediate-term mortality after cardiac surgery in the B-Unaware Trial. Anesthesiology 2010;112:1116-27.

32. Kertai MD, Palanca BJ, Pal N, et al. Bispectral index monitoring, duration of bispectral index below 45 , patient risk factors, and intermediate-term mortality after noncardiac surgery in the B-Unaware Trial. Anesthesiology 2011;114:545-56.

33. Monk TG, Saini V, Weldon BC, et al. Anesthetic management and one-year mortality after noncardiac surgery. Anesth Analg 2005;100:4-10.

34. Lindholm ML, Traff S, Granath F, et al. Mortality within 2 years after surgery in relation to low intraoperative bispectral index values and preexisting malignant disease. Anesth Analg 2009;108:508-12.

35. Leslie K, Myles PS, Forbes A, et al. The effect of bispectral index monitoring on long-term survival in the B-aware trial. Anesth Analg 2010;110:816-22.

36. Willingham M, Ben Abdallah A, Gradwohl S, et al. The association between intraoperative electroencephalographic suppression and postoperative mortality. Br J Anaesth 2014;113:1001-8.

37. National Clinical Guideline Centre (UK). Depth of anaesthesia monitors-bispectral index (BIS), E-Entropy and

Narcotrend-Compact M. London: Royal College of Physicians, 2012.

38. Pandit JJ, Cook TM. National Institute for Clinical Excellence guidance on measuring depth of anaesthesia: limitations of EEG-based technology. Br J Anaesth 2013;110:325-8.

39. Pandit JJ, Cook TM, Jonker WR, et al., 5th National Audit Project of the Royal College of Anaesthetists and the Association of Anaesthetists of Great Britain Ireland. A national survey of anaesthetists (NAP5 baseline) to estimate an annual incidence of accidental awareness during general anaesthesia in the UK. $\mathrm{Br} J$ Anaesth 2013;110:501-9.

40. Avidan MS, Zhang L, Burnside BA, et al. Anesthesia awareness and the bispectral index. N Engl J Med 2008;358:1097-108.

41. Avidan MS, Jacobsohn E, Glick D, et al. Prevention of intraoperative awareness in a high-risk surgical population. $N$ Engl J Med 2011;365:591-600.

42. Mashour GA, Shanks A, Tremper KK, et al. Prevention of intraoperative awareness with explicit recall in an unselected surgical population: a randomized comparative effectiveness trial. Anesthesiology 2012;117:717-25.

43. American Society of Anesthesiologists Task Force on Intraoperative Awareness. Practice advisory for intraoperative awareness and brain function monitoring: a report by the American society of anesthesiologists task force on intraoperative awareness. Anesthesiology 2006;104:847-64.

44. Crosby G. General anesthesia-minding the mind during surgery. N Engl J Med 2011;365:660-1.

45. Morimoto $\mathrm{Y}$, Hagihira S, Koizumi $\mathrm{Y}$, et al. The relationship between bispectral index and electroencephalographic parameters during isoflurane anesthesia. Anesth Analg 2004;98:1336-40, table of contents.

46. Kertai MD, Whitlock EL, Avidan MS. Brain monitoring with electroencephalography and the electroencephalogram-derived bispectral index during cardiac surgery. Anesth Analg 2012;114:533-46.

47. Soehle M, Dittmann A, Ellerkmann RK, et al. Intraoperative burst suppression is associated with postoperative delirium following cardiac surgery: a prospective, observational study. BMC Anesthesiol 2015;15:61.

48. Andresen JM, Girard TD, Pandharipande PP, et al. Burst suppression on processed electroencephalography as a predictor of postcoma delirium in mechanically ventilated ICU patients. Crit Care Med 2014;42:2244-51.

49. Watson PL, Shintani AK, Tyson R, et al. Presence of electroencephalogram burst suppression in sedated, critically il patients is associated with increased mortality. Crit Care Med 2008:36:3171-7.

50. Whitlock EL, Villafranca AJ, Lin N, et al. Relationship between bispectral index values and volatile anesthetic concentrations during the maintenance phase of anesthesia in the B-Unaware trial. Anesthesiology 2011;115:1209-18.

51. Chan AW, Tetzlaff JM, Altman DG, et al. SPIRIT 2013 statement: defining standard protocol items for clinical trials. Ann Intern Med 2013;158:200-7

52. Chan AW, Tetzlaff JM, Gotzsche PC, et al. SPIRIT 2013 explanation and elaboration: guidance for protocols of clinical trials. BMJ 2013;346:e7586

53. Aranake A, Gradwohl S, Ben-Abdallah A et al. Increased risk of intraoperative awareness in patients with a history of awareness. Anesthesiology 2013;119:1275-83.

54. Thabane L, Ma J, Chu R, et al. A tutorial on pilot studies: the what, why and how. BMC Med Res Methodol 2010;10:1.

55. Bottros MM, Palanca BJ, Mashour GA, et al. Estimation of the bispectral index by anesthesiologists: an inverse turing test. Anesthesiology 2011;114:1093-101.

56. ICETAP Electroencephalography Education Website. http://www. icetap.org/ (accessed 31 May 2015).

57. McDowell JA, Mion LC, Lydon TJ, et al. A nonpharmacologic sleep protocol for hospitalized older patients. J Am Geriatr Soc 1998;46:700-5. 
58. Inouye SK, Bogardus ST Jr, Williams CS, et al. The role of adherence on the effectiveness of nonpharmacologic interventions: evidence from the delirium prevention trial. Arch Intern Med 2003;163:958-64.

59. Hospital Elder Life Program: Information About Delirium. http:// www.hospitalelderlifeprogram.org/for-family-members/ about-delirium/ (accessed 31 Oct 2014)

60. Hospital Elder Life Program: Advice on Delirium Management. http://www.hospitalelderlifeprogram.org/for-family-members/ what-you-can-do/ (accessed 31 Oct 2014).

61. Lenze EJ, Host HH, Hildebrand MW, et al. Enhanced medical rehabilitation increases therapy intensity and engagement and improves functional outcomes in postacute rehabilitation of older adults: a randomized-controlled trial. J Am Med Dir Assoc 2012;13:708-12.

62. Ganz DA, Huang C, Saliba D, et al. Preventing falls in hospitals: a toolkit for improving quality of care. (Prepared by RAND Corporation, Boston University School of Public Health, and ECRI Institute under Contract No. HHSA290201000017I TO \#1). Rockville, MD: Agency for Healthcare Research and Quality, 2013. AHRQ Publication No. 13-0015-EF.

63. Rhalimi M, Helou R, Jaecker P. Medication use and increased risk of falls in hospitalized elderly patients: a retrospective, case-contro study. Drugs Aging 2009;26:847-52.

64. Masud T, Frost M, Ryg J, et al. Central nervous system medications and falls risk in men aged $60-75$ years: the Study on Male Osteoporosis and Aging (SOMA). Age Ageing 2013;42:121-4.

65. Olazaran J, Valle D, Serra JA, et al. Psychotropic medications and falls in nursing homes: a cross-sectional study. J Am Med Dir Assoc 2013;14:213-17.

66. van Strien AM, Koek HL, van Marum RJ, et al. Psychotropic medications, including short acting benzodiazepines, strongly increase the frequency of falls in elderly. Maturitas 2013;74:357-62.

67. Payne RA, Abel GA, Simpson CR, et al. Association between prescribing of cardiovascular and psychotropic medications and hospital admission for falls or fractures. Drugs Aging 2013;30:247-54.

68. Askari M, Eslami S, Scheffer AC, et al. Different risk-increasing drugs in recurrent versus single fallers: are recurrent fallers a distinct population? Drugs Aging 2013;30:845-51.

69. Freeland KN, Thompson AN, Zhao Y, et al. Medication use and associated risk of falling in a geriatric outpatient population. Ann Pharmacother 2012:46:1188-92.

70. American Geriatrics Society Beers Criteria Update Expert Panel. American Geriatrics Society updated Beers Criteria for potentially inappropriate medication use in older adults. J Am Geriatr Soc 2012;60:616-31.

71. Carpenter CR, Avidan MS, Wildes T, et al. Predicting geriatric falls following an episode of emergency department care: a systematic review. Acad Emerg Med 2014;21:1069-82.

72. DeCrane SK, Culp KR, Wakefield B. Twelve-month fall outcomes among delirium subtypes. J Healthc Qual 2012;34:13-20.

73. Lakatos BE, Capasso V, Mitchell MT, et al. Falls in the general hospital: association with delirium, advanced age, and specific surgical procedures. Psychosomatics 2009;50:218-26.

74. Siddiqi N, Young J, House AO, et al. Stop Delirium! A complex intervention to prevent delirium in care homes: a mixed-methods feasibility study. Age Ageing 2011;40:90-8.

75. Pautex S, Herrmann FR, Zulian GB. Factors associated with falls in patients with cancer hospitalized for palliative care. J Palliat Med 2008;11:878-84.

76. Mahoney JE, Palta M, Johnson J, et al. Temporal association between hospitalization and rate of falls after discharge. Arch Intern Med 2000;160:2788-95.

77. Harris PA, Taylor R, Thielke R, et al. Research electronic data capture (REDCap)-a metadata-driven methodology and workflow process for providing translational research informatics support. J Biomed Inform 2009;42:377-81.

78. Fritz BA, Rao P, Mashour GA, et al. Postoperative recovery with bispectral index versus anesthetic concentration-guided protocols. Anesthesiology 2013;118:1113-22.

79. Brown CH IV, Morrissey C, Ono M, et al. Impaired olfaction and risk of delirium or cognitive decline after cardiac surgery. J Am Geriatr Soc 2015;63:16-23.

80. Galvin JE, Roe CM, Powlishta KK, et al. The AD8: a brief informant interview to detect dementia. Neurology 2005;65:559-64.

81. Davis PB, Morris JC, Grant E. Brief screening tests versus clinical staging in senile dementia of the Alzheimer type. J Am Geriatr Soc 1990;38:129-35.
82. Weintraub S, Dikmen SS, Heaton RK, et al. The cognition battery of the NIH toolbox for assessment of neurological and behavioral function: validation in an adult sample. J Int Neuropsychol Soc 2014;20:567-78

83. Heaton RK, Akshoomoff N, Tulsky D, et al. Reliability and validity of composite scores from the NIH Toolbox Cognition Battery in adults. $J$ Int Neuropsychol Soc 2014;20:588-98.

84. Rudolph JL, Jones RN, Grande LJ, et al. Impaired executive function is associated with delirium after coronary artery bypass graft surgery. J Am Geriatr Soc 2006;54:937-41.

85. Fong TG, Hshieh TT, Wong B, et al. Neuropsychological profiles of an elderly cohort undergoing elective surgery and the relationship between cognitive performance and delirium. J Am Geriatr Soc 2015;63:977-82.

86. Amrock LG, Deiner $\mathrm{S}$. The implication of frailty on preoperative risk assessment. Curr Opin Anaesthesiol 2014;27: 330-5.

87. Abizanda P, Navarro JL, Garcia-Tomas Ml, et al. Validity and usefulness of hand-held dynamometry for measuring muscle strength in community-dwelling older persons. Arch Gerontol Geriatr 2012;54:21-7.

88. Wei LA, Fearing MA, Sternberg EJ, et al. The confusion assessment method: a systematic review of current usage. J Am Geriatr Soc 2008;56:823-30.

89. Ely EW, Inouye SK, Bernard GR, et al. Delirium in mechanically ventilated patients: validity and reliability of the confusion assessment method for the intensive care unit (CAM-ICU). JAMA 2001;286:2703-10.

90. Ely EW, Margolin R, Francis J, et al. Evaluation of delirium in critically ill patients: validation of the confusion assessment method for the intensive care unit (CAM-ICU). Crit Care Med 2001;29:1370-9.

91. Luetz A, Heymann A, Radtke FM, et al. Different assessment tools for intensive care unit delirium: which score to use? Crit Care Med 2010;38:409-18.

92. Plaschke $\mathrm{K}$, von Haken R, Scholz M, et al. Comparison of the confusion assessment method for the intensive care unit (CAM-ICU) with the intensive care delirium screening checklist (ICDSC) for delirium in critical care patients gives high agreement rate(s). Intensive Care Med 2008;34:431-6.

93. Inouye SK, Leo-Summers L, Zhang Y, et al. A chart-based method for identification of delirium: validation compared with interviewer ratings using the confusion assessment method. J Am Geriatr Soc 2005;53:312-18.

94. Saczynski JS, Kosar CM, Xu G, et al. A tale of two methods: chart and interview methods for identifying delirium. J Am Geriatr Soc 2014;62:518-24.

95. Ramaswamy R, Dix EF, Drew JE, et al. Beyond grand rounds: a comprehensive and sequential intervention to improve identification of delirium. Gerontologist 2011;51:122-31.

96. Sim J, Wright CC. The kappa statistic in reliability studies: use, interpretation, and sample size requirements. Phys Ther 2005;85:257-68.

97. Selim AJ, Rogers W, Fleishman JA, et al. Updated U.S. population standard for the Veterans RAND 12-item Health Survey (VR-12). Qual Life Res 2009;18:43-52.

98. Lamb SE, Jorstad-Stein EC, Hauer K, et al., Prevention of Falls Network Europe, Outcomes Consensus Group. Development of a common outcome data set for fall injury prevention trials: the Prevention of Falls Network Europe consensus. J Am Geriatr Soc 2005;53:1618-22.

99. Skelton DA, Todd CJ, ProFa NEG. Prevention of Falls Network Europe: a thematic network aimed at introducing good practice in effective falls prevention across Europe. Four years on. $\checkmark$ Musculoskelet Neuronal Interact 2007;7:273-8.

100. Tinetti ME, Speechley M, Ginter SF. Risk factors for falls among elderly persons living in the community. $N$ Engl $J$ Med 1988;319:1701-7.

101. Short TG, Leslie K, Campbell D, et al. A pilot study for a prospective, randomized, double-blind trial of the influence of anesthetic depth on long-term outcome. Anesth Analg 2014;118:981-6.

102. Short TG, Leslie K, Chan MT, et al. Rationale and design of the balanced anesthesia study: a prospective randomized clinical trial of two levels of anesthetic depth on patient outcome after major surgery. Anesth Analg 2015;121:357-65.

103. Martins S, Conceicao F, Paiva JA, et al. Delirium recognition by family: European Portuguese validation study of the family confusion assessment method. J Am Geriatr Soc 2014;62:1748-52.

104. Steis MR, Evans L, Hirschman KB, et al. Screening for delirium using family caregivers: convergent validity of the family confusion 
assessment method and interviewer-rated confusion assessment method. J Am Geriatr Soc 2012;60:2121-6.

105. Perez ME, Pericchi LR. Changing statistical significance with the amount of information: the adaptive significance level. Stat Probab Lett 2014;85:20-4.

106. Nuzzo R. Scientific method: statistical errors. Nature 2014:506:150-2.

107. Ioannidis JP. Why most published research findings are false. PLoS Med 2005;2:e124.

108. Prasad V, Vandross A, Toomey C, et al. A decade of reversal: an analysis of 146 contradicted medical practices. Mayo Clin Proc 2013;88:790-8

109. Johnson VE. Revised standards for statistical evidence. Proc Natl Acad Sci USA 2013;110:19313-17.

110. Jones TS, Dunn CL, Wu DS, et al. Relationship between asking an older adult about falls and surgical outcomes. JAMA Surg 2013;148:1132-8.

111. Loudon K, Treweek S, Sullivan F, et al. The PRECIS-2 tool: designing trials that are fit for purpose. BMJ 2015;350:h2147.

112. Glasgow RE, Gaglio B, Bennett G, et al. Applying the PRECIS criteria to describe three effectiveness trials of weight loss in obese patients with comorbid conditions. Health Serv Res 2012;47:1051-67.

113. Thorpe KE, Zwarenstein M, Oxman AD, et al. A pragmatic-explanatory continuum indicator summary (PRECIS): a tool to help trial designers. J Clin Epidemiol 2009;62:464-75.

114. Schuller PJ, Newell S, Strickland PA, et al. Response of bispectra index to neuromuscular block in awake volunteersdagger. $\mathrm{Br} J$ Anaesth 2015;115(Suppl 1):i95-103.

115. Brice DD, Hetherington RR, Utting JE. A simple study of awareness and dreaming during anaesthesia. $\mathrm{Br} J$ Anaesth 1970;42:535-42.

116. Tharmanathan $\mathrm{P}$, Calvert $\mathrm{M}$, Hampton $\mathrm{J}$, et al. The use of interim data and Data Monitoring Committee recommendations in randomized controlled trial reports: frequency, implications and potential sources of bias. BMC Med Res Methodol 2008;8:12.

117. Fleming TR, DeMets DL. Monitoring of clinical trials: issues and recommendations. Control Clin Trials 1993;14:183-97.

118. Smith MA, Ungerleider RS, Korn EL, et al. Role of independent data-monitoring committees in randomized clinical trials sponsored by The National Cancer Institute. J Clin Oncol 1997;15: 2736-43.

119. Montori VM, Devereaux PJ, Adhikari NK, et al. Randomized trials stopped early for benefit: a systematic review. JAMA 2005;294:2203-9

120. Bassler D, Briel M, Montori VM, et al. Stopping randomized trials early for benefit and estimation of treatment effects: systematic review and meta-regression analysis. JAMA 2010;303:1180-7.

121. Grant $A M$, Altman DG, Babiker $A B$, et al. Issues in data monitoring and interim analysis of trials. Health Technol Assess 2005;9:1-238 iii-iv.

122. Myles PS, Leslie K, McNeil J, et al. Bispectral index monitoring to prevent awareness during anaesthesia: the $\mathrm{B}$-Aware randomised controlled trial Lancet 2004:363:1757-63.

123. Mashour GA, Esaki RK, Tremper KK, et al. A novel classification instrument for intraoperative awareness events. Anesth Analg 2010;110:813-15.

124. Bellelli G, Mazzola P, Morandi A, et al. Duration of postoperative delirium is an independent predictor of 6-month mortality in older adults after hip fracture. J Am Geriatr Soc 2014;62:1335-40.

125. Bryczkowski SB, Lopreiato MC, Yonclas PP, et al. Delirium prevention program in the surgical intensive care unit improved the outcomes of older adults. J Surg Res 2014;190:280-8.

126. Morandi A, Rogers BP, Gunther ML, et al. The relationship between delirium duration, White matter integrity, and cognitive impairment in intensive care unit survivors as determined by diffusion tensor imaging: the VISIONS prospective cohort magnetic resonance imaging study*. Crit Care Med 2012;40:2182-9.
127. Gunther ML, Morandi A, Krauskopf E, et al. The association between brain volumes, delirium duration, and cognitive outcomes in intensive care unit survivors: the VISIONS cohort magnetic resonance imaging study. Crit Care Med 2012;40:2022-32.

128. Inouye SK, Kosar CM, Tommet D, et al. The CAM-S: development and validation of a new scoring system for delirium severity in 2 cohorts. Ann Intern Med 2014;160:526-33.

129. Avidan MS, Fritz BA, Maybrier HR, et al. The Prevention of Delirium and Complications Associated with Surgical Treatments (PODCAST) study: protocol for an international multicentre randomised controlled trial. BMJ Open 2014;4:e005651.

130. Chanques G, Payen JF, Mercier G, et al. Assessing pain in non-intubated critically ill patients unable to self report: an adaptation of the Behavioral Pain Scale. Intensive Care Med 2009;35:2060-7.

131. Jacobson SA, Dwyer PC, Machan JT, et al. Quantitative analysis of rest-activity patterns in elderly postoperative patients with delirium: support for a theory of pathologic wakefulness. J Clin Sleep Med 2008;4:137-42.

132. van Dellen E, van der Kooi AW, Numan T, et al. Decreased functional connectivity and disturbed directionality of information flow in the electroencephalography of intensive care unit patients with delirium after cardiac surgery. Anesthesiology 2014;121:328-35.

133. van der Kooi AW, Zaal IJ, Klijn FA, et al. Delirium detection using EEG: what and how to measure? Chest 2015;147:94-101.

134. Hofste WJ, Linssen CA, Boezeman EH, et al. Delirium and cognitive disorders after cardiac operations: relationship to pre- and intraoperative quantitative electroencephalogram. Int J Clin Monit Comput 1997;14:29-36.

135. Pollak CP, Tryon WW, Nagaraja $\mathrm{H}$, et al. How accurately does wrist actigraphy identify the states of sleep and wakefulness? Sleep 2001;24:957-65.

136. Charlson ME, Pompei $\mathrm{P}$, Ales KL, et al. A new method of classifying prognostic comorbidity in longitudinal studies: development and validation. J Chronic Dis 1987;40:373-83.

137. Kocalevent RD, Hinz A, Brahler E. Standardization of the depression screener patient health questionnaire (PHQ-9) in the general population. Gen Hosp Psychiatry 2013;35:551-5.

138. Payen JF, Bru O, Bosson JL, et al. Assessing pain in critically ill sedated patients by using a behavioral pain scale. Crit Care Med 2001;29:2258-63.

139. Takeuchi M, Takeuchi $H$, Fujisawa $D$, et al. Incidence and risk factors of postoperative delirium in patients with esophageal cancer. Ann Surg Oncol 2012;19:3963-70.

140. Chang YL, Tsai YF, Lin PJ, et al. Prevalence and risk factors for postoperative delirium in a cardiovascular intensive care unit. $A m \mathrm{~J}$ Crit Care 2008;17:567-75

141. Smulter N, Lingehall HC, Gustafson Y, et al. Delirium after cardiac surgery: incidence and risk factors. Interact Cardiovasc Thorac Surg 2013;17:790-6.

142. Guenther U, Theuerkauf N, Frommann I, et al. Predisposing and precipitating factors of delirium after cardiac surgery: a prospective observational cohort study. Ann Surg 2013;257:1160-7.

143. Bakker RC, Osse RJ, Tulen JH, et al. Preoperative and operative predictors of delirium after cardiac surgery in elderly patients. Eur $J$ Cardiothorac Surg 2012;41:544-9.

144. Rudolph JL, Jones RN, Levkoff SE, et al. Derivation and validation of a preoperative prediction rule for delirium after cardiac surgery. Circulation 2009;119:229-36.

145. Marcantonio ER, Goldman L, Mangione CM, et al. A clinical prediction rule for delirium after elective noncardiac surgery. JAMA 1994:271:134-9.

146. Greene NH, Attix DK, Weldon BC, et al. Measures of executive function and depression identify patients at risk for postoperative delirium. Anesthesiology 2009;110:788-95.

147. Flink BJ, Rivelli SK, Cox EA, et al. Obstructive sleep apnea and incidence of postoperative delirium after elective knee replacement in the nondemented elderly. Anesthesiology 2012;116:788-96. 
Correction: Protocol for the Electroencephalography Guidance of Anesthesia to Alleviate Geriatric Syndromes (ENGAGES) study: a pragmatic, randomised clinical trial

Wildes TS, Winter AC, Maybrier HR, et al. Protocol for the Electroencephalography Guidance of Anesthesia to Alleviate Geriatric Syndromes (ENGAGES) study: a pragmatic, randomised clinical trial. BMJ Open 2016;6:e011505.

Two authors' names are incorrect in this manuscript. 'J Leung' should be cited as 'JM Leung' and 'RL Hueneke' should be cited as 'R Hueneke.'

Open Access This is an Open Access article distributed in accordance with the Creative Commons Attribution Non Commercial (CC BY-NC 4.0) license, which permits others to distribute, remix, adapt, build upon this work non-commercially, and license their derivative works on different terms, provided the original work is properly cited and the use is non-commercial. See: http://creativecommons.org/licenses/by-nc/4.0/

BMJ Open 2016;6:e011505corr1. doi:10.1136/bmjopen-2016-011505corr1 